\title{
Narrowly Confined and Glomerulus-Specific Onset Latencies of Odor-Evoked Calcium Transients in the Juxtaglomerular Cells of the Mouse Main Olfactory Bulb
}

\author{
Ryota Homma, ${ }^{{ }^{*}}$ Xiaohua Lv, ${ }^{1,2,3^{*}}$ Tokiharu Sato, ${ }^{1}$ Fumiaki Imamura, ${ }^{4}$ Shaoqun Zeng, ${ }^{2,3}$ and Shin \\ Nagayama ${ }^{1}$
}

https://doi.org/10.1523/ENEURO.0387-18.2019

\begin{abstract}
${ }^{1}$ Department of Neurobiology and Anatomy, McGovern Medical School at the University of Texas Health Science Center at Houston, Houston, Texas 77030, ${ }^{2}$ Britton Chance Center for Biomedical Photonics, Huazhong University of Science and Technology, Wuhan 430074, China, ${ }^{3} \mathrm{MoE}$ Key Laboratory for Biomedical Photonics, Collaborative Innovation Center for Biomedical Engineering, School of Engineering Sciences, Huazhong University of Science and Technology, Wuhan 430074, China, and ${ }^{4}$ Department of Pharmacology, Pennsylvania State University College of Medicine, Hershey, Pennsylvania 17033
\end{abstract}

\begin{abstract}
Odor information is transmitted from olfactory sensory neurons to principal neurons at the glomeruli of the olfactory bulb. The intraglomerular neuronal circuit also includes hundreds of interneurons referred to as juxtaglomerular (JG) cells. Stimulus selectivity is well correlated among many JG cells that are associated with the same glomerulus, consistent with their highly homogeneous sensory inputs. However, much less is known about the temporal aspects of their activity, including the temporal coordination of their odor-evoked responses. As many JG cells within a glomerular module respond to the same stimulus, the extent to which their activity is temporally aligned will affect the temporal profile of their population inhibitory inputs. Using random-access high-speed two-photon microscopy, we recorded the odor-evoked calcium transients of mouse JG cells and compared the onset latency and rise time among neurons putatively associated with the same and different glomeruli. Whereas the overall onset latencies of odorevoked transients were distributed across a $\sim 150 \mathrm{~ms}$ time window, those from cells putatively associated with the same glomerulus were confined to a much narrower window of several tens of milliseconds. This result suggests that onset latency primarily depends on the associated glomerulus. We also observed glomerular specificity in the rise time. The glomerulus-specific temporal pattern of odor-evoked activity implies that the temporal patterns of inputs from the intraglomerular circuit are unique to individual glomerulus-odor pairs, which may contribute to efficient shaping of the temporal pattern of activity in the principal neurons.
\end{abstract}

Key words: AOD; glomerulus; respiration; two-photon

\section{Significance Statement}

The sense of smell is essential for assessing chemicals in one's atmospheric environment. In understanding the biological mechanisms by which humans and animals recognize various odorous chemicals, a key question is how the signals sent from the nose are transformed in the brain. In this work, we studied the temporal pattern of activity in interneurons that tune the sensory inputs to the principal (signal-carrying) neurons in the olfactory bulb via structures known as glomeruli. We found that the odor responses of these interneurons are precisely coordinated in time, suggesting that the glomerulus is a temporally wellorganized unit. Our finding suggests that the temporal coordination of glomerular activity is important in signal transformation in the olfactory system. 


\section{Introduction}

The glomeruli in the olfactory bulb (OB) form an attractive model system for studying signal processing owing to their well-documented neurons and characteristic anatomy, in which the input and output pathways are well segregated. In a glomerulus, sensory inputs from olfactory sensory neurons (OSNs) are transmitted to mitral/ tufted cells, which in turn send the signals to higher olfactory centers. Signal processing in the glomerulus involves feedforward excitation and inhibition mediated by hundreds of juxtaglomerular (JG) cells, the diverse interneurons (next paragraph) located in the glomerular layer (GL; De Saint Jan et al., 2009; Najac et al., 2011; Shao et al., 2012; Carey et al., 2015; Geramita and Urban, 2017). One remarkable trait of these feedforward circuits is that each type of feedforward input (excitatory or inhibitory) to a glomerulus is mediated by numerous JG cells of specific types. The properties of collective feedforward inputs thus depend on the extent to which the activity of participating neurons is temporally coordinated. However, little is known about the temporal patterns of activity among JG cells that are associated with the same glomerulus.

The term "JG cell" is a generic name for interneurons in the $\mathrm{GL}$ and includes multiple types of neurons: conventionally, major JG cell types include GABAergic periglomerular (PG) cells, glutamatergic external tufted (ET) cells, and GABAergic/dopaminergic superficial short-axon (sSA) cells (Pinching and Powell, 1971; Nagayama et al., 2014; but see Tavakoli et al., 2018). PG cells are the most abundant cell type among JG cells. They are monoglomerular (their dendrites project to a single glomerulus) and they receive sensory inputs directly from OSNs or disynaptically from ET cells, depending on the PG cell subtype (Shao et al., 2009). ET cells are also monoglomerular and receive direct input from OSNs. These two cell types are the sources of feedforward inhibition and excitation, respectively, in the intraglomerular circuit through multiple subcircuits (Shao et al., 2009; Najac et al., 2015). The neuronal processes of sSA cells cross multiple glomeruli and are believed to mediate interactions among glomeruli within the GL (Kiyokage et al., 2010). The cellular properties and connectivity of these neurons, including their

Received October 2, 2018; accepted February 5, 2019; First published February 11, 2019.

The authors declare no competing financial interests.

Author contributions: R.H. and S.N. designed research; R.H., X.L., T.S., F.I.,

S.Z., and S.N. performed research; R.H. analyzed data; R.H. and S.N. wrote the paper.

This work was supported by the $\mathrm{NIH}$, National Institute on Deafness and Other Communication Disorders (Grants R01DC013802 and R01DC016307) and National Natural Science Foundation of China (Grants 81327802 and 61721092). We thank Wei R. Chen for his contribution to the setup of the acousto-optic deflector two-photon microscope.

${ }^{*} \mathrm{RH}$ and $\mathrm{XL}$ contributed equally to this work.

Correspondence should be addressed to Shin Nagayama at Shin.Nagayama@ uth.tmc.edu or Ryota Homma at Ryota.Homma@uth.tmc.edu.

https://doi.org/10.1523/ENEURO.0387-18.2019

Copyright @ 2019 Homma et al.

This is an open-access article distributed under the terms of the Creative Commons Attribution 4.0 International license, which permits unrestricted use, distribution and reproduction in any medium provided that the original work is properly attributed. subtypes, have been extensively studied (Wachowiak and Shipley, 2006; Kosaka and Kosaka 2014, 2016; Burton 2017; Tavakoli et al., 2018).

The basic functional properties of JG cells have been revealed previously by in vivo recordings (Wellis and Scott, 1990; Tan et al., 2010; Homma et al., 2013; Kikuta et al., 2013; Wachowiak et al., 2013; Fukunaga et al., 2014; Livneh et al., 2014). For example, JG cells fire spontaneously at various rates; this spontaneous activity is phasic and tuned to respiration phase in the majority of cells. In response to odors, JG cell firing increases or decreases and/or shifts in phase. The relationship between glomerular inputs and JG cells has been studied via electrophysiological recordings in genetically tagged glomeruli (Tan et al., 2010), calcium imaging with glomerulus-specific labeling (Kikuta et al., 2013), and calcium imaging with optogenetic stimulation of a genetically-targeted single glomerulus (Braubach et al., 2018). The odor selectivity of JG cells is correlated with that of OSNs as well as with that of other JG cells associated with the same glomerulus. However, these techniques did not allow simultaneous recording from multiple JG cells at sufficient temporal resolution to analyze how the activity of these cells is temporally coordinated. In this study, we used high-speed two-photon calcium recording (Grewe et al., 2010) to compare the calcium transients in JG cells within and across glomeruli at a high temporal resolution. We demonstrated that the time course of odorevoked calcium transients is primarily determined by the glomerulus. The onset latency of JG cells was highly heterogeneous, with a $\sim 150 \mathrm{~ms}$ difference between the earliest and the latest responses, but onset latency was confined to a much narrower window when we considered only the cells putatively associated with the same glomerulus. Such coordinated activity in JG cells could help to efficiently shape the time course of sensory inputs that are unique to the associated glomerulus.

\section{Materials and Methods}

\section{Materials}

Eight mice ( 1 female) that were the progeny of a Gad2IRES-Cre mouse (Taniguchi et al., 2011; JAX stock \#10802; RRID:IMSR_JAX:010802) and a cre-recombinase-dependent tdTomato reporter mouse (Ai9; Madisen et al., 2010; JAX stock \#7909; RRID:IMSR_JAX:007909) were used in this study. An adeno-associated virus (AAV) vector that encodes the GCaMP6f gene under the synapsin promoter (AAV1.Syn.GCaMP6f.WPRE.SV40) was purchased from the UPenn Vector Core. All odorants were purchased from Sigma-Aldrich.

\section{Animal preparation}

All animal procedures were conducted in accordance with an animal protocol that was approved by the Institutional Animal Care and Use Committee (IACUC) of The University of Texas Health Science Center at Houston (UTHealth).

\section{Viral injections}

Animals were anesthetized with an intraperitoneal injection of ketamine/xylazine $(10 / 0.5 \mathrm{mg} / \mathrm{ml} \mathrm{k} / \mathrm{x}, 12 \mu \mathrm{l} / \mathrm{g}$ body- 
weight). The depth of anesthesia was routinely monitored by toe pinches, and additional injections of anesthetic were made to maintain the appropriate depth of anesthesia. Rectal body temperature was maintained between $36.0^{\circ} \mathrm{C}$ and $37.0^{\circ} \mathrm{C}$. The skull above the dorsal OBs was exposed, and two small holes corresponding to two injection sites were made above the posterior end of one $\mathrm{OB}$. For each injection, a glass pipette containing the AAV suspension (no dilution: $9 \times 10^{12} \mathrm{GC} / \mathrm{ml}$ ) was inserted through one of the holes. The pipette approached from the posterior side of the animal, parallel to the anteriorposterior axis and tilted $30^{\circ}-45^{\circ}$ from vertical. The tip of the pipette was advanced 250-300 $\mu \mathrm{m}$ from the bulbar surface. A 320-640 $\mathrm{nl}$ injection was made at the rate of 64-128 $\mathrm{nl} / \mathrm{min}$ with a Nanoject II oil-pressure injector (Drummond) in each of the two injection sites. After surgery, mice were left to recover undisturbed for 15-34 d before the recording session. With this injection protocol, uniform labeling of the entire dorsal $\mathrm{OB}$ was observed under a fluorescence microscope. In some of the animals (labeled Mice 6-8 in the figures), a cranial window was implanted during the surgery for the AAV injections. In these animals, a metal headplate with a $5 \mathrm{~mm}$ round opening (CP-1, Narishige) was attached to the skull with cyanoacrylate glue and dental acrylic. A round cranial window ( $\sim 3 \mathrm{~mm}$ diameter) that covered both dorsal bulbs was made through the opening of the headplate. The dura mater was not removed. The exposed bulb was sealed with $0.8 \%$ agarose and a $3 \mathrm{~mm}$ round coverslip (CS-3R, Warner Instruments), which was cemented with cyanoacrylate glue and dental acrylic.

\section{Data acquisition}

Animals previously injected with AAV were anesthetized with an intraperitoneal injection of urethane $(6 \% \mathrm{w} / \mathrm{v}, 20$ $\mu \mathrm{l} / \mathrm{g}$ bodyweight for induction, with the depth of anesthesia monitored by toe pinches and maintained by additional injections). Animals breathed freely throughout the experiment, and rectal body temperature was maintained between $36.0^{\circ} \mathrm{C}$ and $37.0^{\circ} \mathrm{C}$. Animals with a pre-installed cranial window (see Viral injections above) were transferred to the microscope stage once the level of anesthesia was stable. For animals without a pre-installed cranial window, a metal headplate was attached to the skull with cyanoacrylate glue and dental acrylic. Then, a craniotomy was made in the AAV-injected side of the $\mathrm{OB}$ and the opening was filled with $1.2 \%$ agarose and sealed with a pre-cut piece of coverslip, which was cemented with cyanoacrylate glue. Following the cranial window preparation, the animal was transferred to the microscope stage. The microscope stage was equipped with an angle-adjustable stage (MAG-3, Narishige) to allow fine adjustments of the angle of the bulbar surface relative to the objective. Breathing was monitored with a piezoelectric sensor placed beneath the chest, and the signal was recorded together with the optical signals.

\section{Optical recordings}

All optical recordings (i.e., wide-field and two-photon) were conducted with the same upright fluorescence microscope (Olympus) combined with a motorized stage
(Scientifica). All optics were implemented on a vibrationisolating air table (Newport). For wide-field imaging, the excitation light was provided by a $470 \mathrm{~nm}$ LED module (M470L2, Thorlabs). The filter set was a standard GFP cube (GFP-4050A-OMF-ZERO, Semrock; 466/495/525 $\mathrm{nm}$ exciter/DM/emitter). A $10 \times / 0.3 \mathrm{NA}$ objective lens (Olympus) and a $0.35 \times$ tube lens (Olympus) were used, and the images were captured by a high-speed CCD camera (NeuroCCD-SM256, RedShirtlmaging) at $125 \mathrm{~Hz}$ for $12 \mathrm{~s}$. With this configuration, a $1.75 \times 1.75 \mathrm{~mm}^{2}$ area of bulbar surface was imaged at $128 \times 128$ pixels. For two-photon recordings, a custom-built acousto-optic deflector (AOD)-based two-photon scanner that is capable of random-access recording was used (lyer et al., 2006; Grewe et al., 2010). Random-access scanning is a mode of two-photon recording that allows one to record from only an arbitrary set of predesignated pixels in the fieldof-view, typically at a much higher sampling rate than conventional full-field imaging. A random-access pattern scanning mode was adopted (Grewe et al., 2010), in which a fixed set of adjacent pixels were also sampled in every sampling cycle so that the total light exposure time for each pixel was reduced. The basic design of the AOD two-photon scanner is adopted from Lv et al. (2006). The light source was a Ti:sapphire laser (MaiTai HP DS, Spectra-Physics), and $920 \mathrm{~nm}$ light was used for all twophoton recordings. The emitted fluorescence was routed via a dichroic mirror (FF665-Di02, Semrock) to the detector module, which was located adjacent to the filter cube to minimize the length of the light path. The detector module contained an IR-block filter (FF01-680/SP, Semrock) as well as a $562 \mathrm{~nm}$ dichroic mirror (FF562-Di02, Semrock) that separates shorter (green) and longer (red) wavelength light. The separated shorter and longer wavelengths of light were detected by a GaAsP photosensor module (H7422A-40, Hamamatsu) and a photomultiplier tube (R1924A, Hamamatsu), respectively. Full-frame scan recordings for functional imaging were made at $2.1 \mathrm{~Hz}$ for $15 \mathrm{~s}$ with a resolution of $217 \times 217$ pixels. With the Olympus $25 \times / 1.05$ NA objective lens that used in this study, the field-of-view was $219 \times 219 \mu \mathrm{m}^{2}$. For the high-speed random-access recordings, 15 sites were predetermined on the basis of the odor-response data obtained during the frame scan recordings (Figs. $2 B, 3 B$, $4 B$ ). Because of relatively low fluorescence intensity of GCaMP6f in the prestimulus period, some of the cells were identifiable only during odor-evoked responses. Although the system is capable of recording a larger number of sites (at a lower sampling rate), 15 was about the maximum number of cells with clear odor-evoked responses that could be identified from a given field-ofview. Therefore, all random-access recordings presented in this study were conducted with 15 sites, each of which covered a single neuron, at a sampling rate of $667 \mathrm{~Hz}$ for $15 \mathrm{~s}$.

\section{Stimulus presentation}

Odor stimuli were presented via a custom-built olfactometer. Briefly, a mixture of odorous gas (diluted odorant bubbled with $100 \%$ nitrogen) at $100 \mathrm{ml} / \mathrm{min}$ and carrier 
gas (filtered air) at $400 \mathrm{ml} / \mathrm{min}$ was continuously supplied but continuously vacuumed near the tip of the applicator. The vacuum was suspended with an electric command signal, resulting in the presentation of odorous gas until the vacuum resumed. In a test conducted with a photoionic odorant sensor (mini PID model 200B, Aurora Scientific), the onset and offset of actual odorant delivery was delayed by $\sim 100 \mathrm{~ms}$ relative to the timing of the command signals. The olfactometer was controlled by a custom-written program in LabVIEW (National Instruments; RRID:SCR_014325) that enabled the stimulus presentation to be synchronized to the respiration cycle of the mouse. Four seconds after the onset of data acquisition, the command signal for an odor stimulus was sent at the peak of the first respiration signal (corresponding to the end of an inhalation cycle) so that the odorant did not reach the mouse in the middle of an inhalation. The duration of the stimulus was either $2 \mathrm{~s}$ or a single respiration cycle, in which case the command signal to terminate the odor stimulus was sent at the peak of the respiration signal in the immediately subsequent cycle.

For the odor stimuli, five single-molecule odorants that were aliphatic aldehydes with carbon chain lengths of three to seven (referred to as $3-7 \mathrm{CHO}$, respectively) were used. All odorants were diluted 1:100 with mineral oil and further flow-diluted 1:5 at the olfactometer. Therefore, the nominal final concentration of the odorants was $0.2 \%$. Five odorants $(3-7 \mathrm{CHO})$ were presented in a block design, with each odorant presented once in a block of five trials. For Mice 1-5, the odorant presentation order was the same in every block. For Mice 6-8, the order was pseudorandomized with the constraint that the same odorant was not presented in two sequential trials. In alternate blocks, the stimulus was presented for $2 \mathrm{~s}$ or for a single respiration cycle.

\section{Immunohistochemistry}

Mice were deeply anesthetized and fixed by transcardial perfusion with $4 \%$ paraformaldehyde in $0.1 \mathrm{~m}$ sodium phosphate buffer, pH 7.4. Then, brain samples were collected and postfixed overnight at $4^{\circ} \mathrm{C}$. The samples were cryoprotected in $30 \%$ sucrose (w/v) in PBS, $\mathrm{pH} 7.4$, and embedded in optimal cutting temperature compound (Sakura Finteck). The olfactory tissues were cut on a cryostat into $20 \mu \mathrm{m}$ sections (coronal) and stored at $-20^{\circ} \mathrm{C}$ until use. The slices were pretreated in TBS-T [10 $\mathrm{mm}$ Tris-HCl, $\mathrm{pH} 7.4,100 \mathrm{~mm} \mathrm{NaCl}$ with $0.3 \%$ Triton X-100 $(\mathrm{v} / \mathrm{v})]$ for $15 \mathrm{~min}$ and blocked with blocking buffer [5\% normal donkey serum (v/v) in TBS-T] at $20-25^{\circ} \mathrm{C}$ for $1 \mathrm{~h}$. The slices were then incubated with primary antibodies diluted in blocking buffer overnight at $4^{\circ} \mathrm{C}$. Sections were washed with TBS-T, and then incubated with secondary antibodies for $1 \mathrm{~h}$. The immunoreacted sections were washed and mounted with FLUORO-GEL mounting medium (Electron Microscopy Sciences, 17985-11).

The following antibodies were used. Primary antibodies: anti-GABA (MilliporeSigma, A2052; rabbit, 1:100) and anti-TH (MilliporeSigma, AB152; rabbit, 1:500). Secondary antibody: AlexaFluor 488 donkey anti-rabbit IgG (ThermoFisher Scientific, A21206; 1:300).
Six to eight serial images were captured at intervals of $1.5 \mu \mathrm{m}$ with an Olympus FluoView FV1000 laser scanning confocal microscope with a $20 \times$ objective lens (UPLSAPO $20 \times$ ), with or without $4 \times$ digital zoom. Then, the acquired images were converted to a Z-stack with the software FV10-ASW viewer (Olympus).

\section{Data analysis}

There were minor differences in the experimental conditions (e.g., the timing of the craniotomy and the order of stimulus presentation; see the sections Animal preparation and Stimulus presentation) between the two subsets of mice (Mice $1-5$ vs $6-8$ ). Because no difference was noticed between the data from these two subsets, they were not discriminated in any of the following analyses. All data analyses and preparation of figures were conducted in Fiji/ImageJ (Schindelin et al., 2012; RRID:SCR_002285) and MATLAB (MathWorks, RRID:SCR_001622) with custom scripts. In the recordings in this study, photobleaching of fluorescence within a trial was negligibly small compared with the evoked responses with which the onset latency and rise time were analyzed. However, during the process of grouping cells based on the stimulus selectivity (see Response-based grouping of cells below), photobleaching was not negligible for the subset of trials that showed little or no odor-evoked response. (Such trials were not included in the subsequent analyses.) Therefore, the photobleaching was corrected for during the process of grouping, but the uncorrected data were used in the rest of analyses because no considerable benefit of correction was recognized.

\section{Spatiotemporal activity maps}

For wide-field imaging, spatial patterns of odor-evoked activity were visualized as the difference between images from the prestimulus period and the response period (i.e., $\Delta F / F_{0}$ maps; Fig. $2 A$ ). A baseline image from the prestimulus period was obtained by averaging the frames from 3 to $4 \mathrm{~s}$ after acquisition onset. Images for three different response periods (early, middle, and late) were obtained by averaging the frames in the $2 \mathrm{~s}$ time windows following $4.5,6.5$, or $8.5 \mathrm{~s}$, respectively, from the acquisition onset $(0.5,2.5$, and $4.5 \mathrm{~s}$ from the stimulus onset). In this specific analysis, the trial-by-trial jitter of inhalation onset or stimulus onset was not taken into account. Then, the prestimulus-period image was subtracted from each of three response-period images. The subtracted images were divided by a spatially filtered prestimulus-period image (two passes of a $3 \times 3$ mean filter) for normalization. After the division, the resulting image was also filtered by two passes of a $3 \times 3$ mean filter. Red, green, and blue color channels were assigned to the resulting images for the early, middle, and late response periods, respectively, and then all three channels were merged to form a full-color map. Therefore, the brightness of the resulting activity maps reflects the response amplitude and the hue reflects the character of the response time course. The intensity scale was set to the same value in each of the three color channels. Essentially the same procedure was applied to obtain the activity maps for two-photon full-frame imaging (Fig. 2B). 


\section{Basic signal processing of the time course data}

Data from two-photon random-access recordings are not a series of images, but a set of time series that resemble the data from multielectrode recordings. Thus, no image processing is involved in the analysis. Time courses were converted to $\Delta F / F_{0}$ by subtracting the averaged data from the first $1 \%$ of data points (150 ms; $F_{0}$ ) and then dividing by $F_{0}$. A box filter with a $100 \mathrm{~ms}$ window was applied to all the time courses unless specified.

\section{Response-based grouping of cells}

The subset of recorded cells that surrounded the same glomerulus had highly correlated odor selectivity (Fig. 3). Under the assumption that these neurons were associated with the same glomerulus, such neurons were grouped using the following two criteria. First, each neuron was directly facing the glomerulus of interest so that no other glomerulus was located between the cell and the glomerulus. When a cell was directly facing more than one glomerulus, all of the facing glomeruli were considered for the second criterion with the other neurons surrounding each glomerulus. Second, the response profile of a given neuron was closer to that of all the in-group recorded neurons than to that of any out-group recorded neurons. In other words, the least similar in-group neuron had a more-similar response profile than the most-similar outgroup neuron. Comparisons of similarity were made simultaneously but not in an agglomerative manner. When a subset of the cells that meet the first criterion did not meet the second, those cells were removed from the group. Cosine similarity (cosine of the angle between two vectors) was used as a measure of the similarity between two response profiles. A response profile here is defined as a vector representing the response amplitudes to the five different odors, or a vector representing all pairwise differences (10 pairs from 5 odorants) of response amplitudes. When at least one of these two response profiles satisfied the above criteria, the neuron was considered as qualified. Response amplitudes were defined as the integral of the $\Delta F / F_{0}$ trace (area under the trace) from the trials with single-respiration-cycle stimulation. In this analysis, because even tiny responses are potentially influential, photobleaching/drift was corrected for by subtracting a straight line that was best fitted to the 0-4 s (prestimulus period) and the 14-14.5 s period (post-response period) of the time course, only when the average of the latter period had a negative value. The correction was made only in the trials with downward changes because (1) prominent photobleaching/drift was observed only in the negative direction, and (2) applying correction to positive values would significantly distort the subset of data that showed long-lasting odor responses.

\section{Determining the onset latency and rise time of calcium transients}

The onset latency of an odor-evoked calcium transient was defined via the following procedure (see also a visual explanation in Fig. $5 A$ ). All $\Delta F / F_{0}$ traces were preprocessed with a $50 \mathrm{~ms}$ box filter. For a given trace, the baseline period was defined as a 100 ms time window immediately before the stimulus onset (the onset of the command signal). The baseline $\Delta F / F_{0}$ level was determined as the mean value over the baseline period. To determine the noise level, the $4 \mathrm{~s}$ prestimulus period was divided into eight $0.5 \mathrm{~s}$ blocks, calculated the standard deviation within each block, and then selected the minimum among these. This procedure effectively eliminated the contribution of the occasional calcium transient (Fig. $4 A$ ) on the noise level. The provisional onset latency was determined as the first time point in which $95 \%$ of the data points in the subsequent $100 \mathrm{~ms}$ exceeded the threshold (2.5 times the noise level). Then, the trace within the subsequent $100 \mathrm{~ms}$ time window was fitted to a straight line. The intersection of the fitted line and the baseline level was considered the onset of the calcium transient. The onset latency was defined as the duration from the onset of the first inhalation with the odor stimulus to the onset of the calcium transient. Although this procedure determined the onset time more accurately than simple threshold methods in high signal-to-noise $(\mathrm{s} / \mathrm{n})$ ratio data, it frequently resulted in obviously wrong values when the $\mathrm{s} / \mathrm{h}$ ratio was lower. Therefore, for this analysis, only data with a relatively high $\mathrm{s} / \mathrm{n}$ ratio were considered. The $\mathrm{s} / \mathrm{n}$ ratio was determined as the slope of the fitted line (in $\Delta F / F_{0}$ per second) divided by the noise level defined above (in $\Delta F / F_{0}$ ). The threshold $\mathrm{s} / \mathrm{n}$ ratio was determined empirically and set to $40 \mathrm{~s}^{-1}$. In addition, in a small fraction of trials, the onset of odor-evoked calcium transients accidentally coincided with a "spontaneous" calcium transient. Such trials were discarded. The estimated onset latency was used for further analysis (Figs. 5-7) when the latency was successfully estimated in three or more trials (of 5-17 trials) for a given cell-odor pair.

The rise time of an odor-evoked transient was defined as the duration between the time points when the signal reached 20 and $80 \%$ of the peak amplitude. For this analysis, all $\Delta F / F_{0}$ traces were preprocessed with a 100 ms box filter. The baseline level was defined in the same way as the protocol for determining onset latency (see above). The peak amplitude was determined as the maximum value of the moving average of a $100 \mathrm{~ms}$ time window. Time points at 20 and $80 \%$ of the peak amplitude were defined as the earliest time point when half of the data points exceeded the threshold value in a $100 \mathrm{~ms}$ time window centered at the time point being considered.

To compare the onset latency and rise time between tdTomato $^{+}$and tdTomato ${ }^{-}$neurons, taking into account their glomerular association, relative onset latency and relative rise time were used. Relative latency, for example, was defined for each group of cells putatively associated with the same glomerulus (including both tdTomato ${ }^{+}$and tdTomato $^{-}$neurons). Subset of groups did not include any tdTomato ${ }^{-}$neurons and thus were excluded from the analysis. The relative latency ranged from zero (shortest) to one (longest), with equal spacing among neurons in the same group. For instance, if a group contained five neurons, their relative latencies would be $0,0.25,0.5,0.75$, and 1, from shortest to longest onset latency. If tdTomato ${ }^{+}$neurons have shorter or longer onset latencies on average, the distribution of relative onset latencies would 


\section{Gad2-ires-Cre x tdTomato reporter (Ai9)}

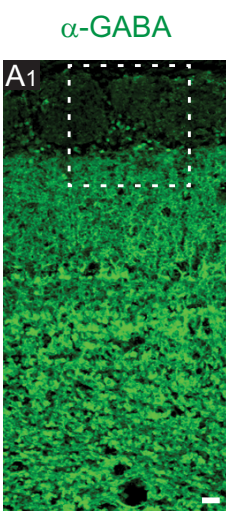

tdTomato
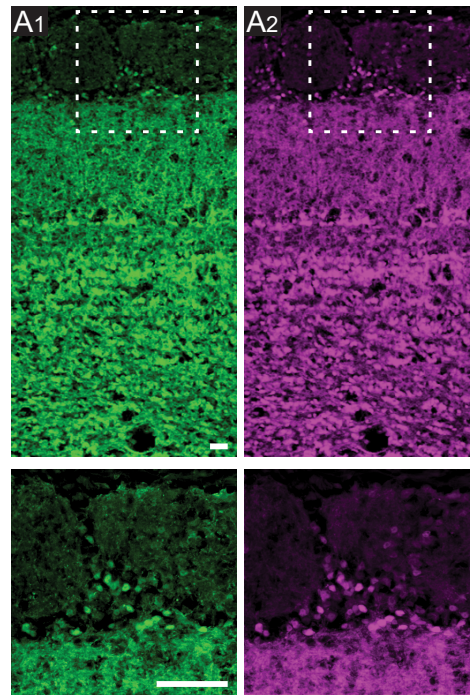

Merge

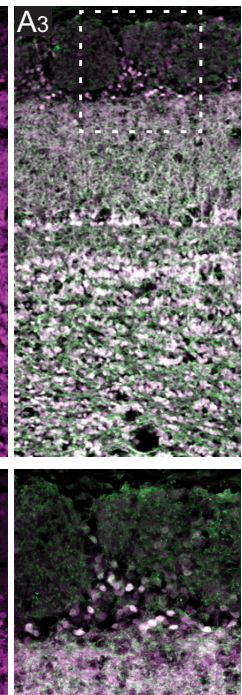

$\alpha-\mathrm{TH}$
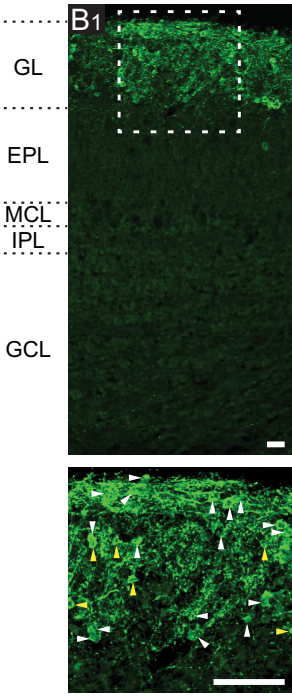

tdTomato
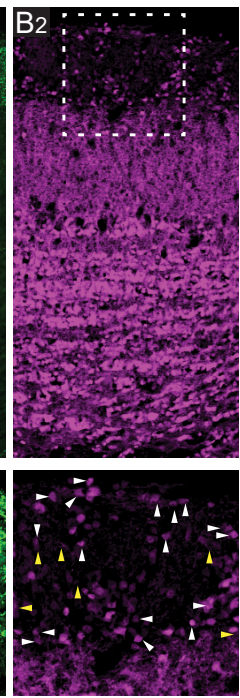

Merge

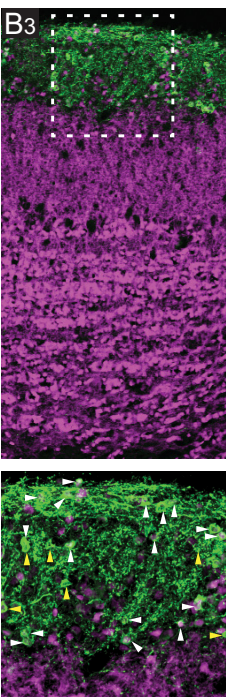

Figure 1. Expression patterns of tdTomato. The progeny of Gad2-IRES-Cre and cre-dependent tdTomato reporter mice were used in this study. $\boldsymbol{A 1 - A 3 , ~ S p a t i a l ~ p a t t e r n ~ o f ~ i m m u n o l a b e l e d ~ G A B A ~ ( A 1 ) , ~ t d T o m a t o ~ ( A 2 ) , ~ a n d ~ t h e ~ m e r g e d ~ i m a g e ~}(\boldsymbol{A} 3)$. Top, Regions within the white boxes are presented at a higher-magnification in the corresponding bottom panels. $\boldsymbol{B} 1-\mathbf{B} 3$, Spatial pattern of immunolabeled TH (B1), tdTomato (B2), and the merged image (B3). White and yellow arrowheads indicate examples of $\mathrm{TH}^{+} / \mathrm{tdTomato}^{+}$cells and $\mathrm{TH}^{+} /$tdTomato $^{-}$cells, respectively. Approximate positions of layer boundaries are indicated by black dotted lines in the space between $\mathbf{A} \mathbf{3}$ and $\boldsymbol{B 1}$. Scale bars, $50 \mu \mathrm{m}$.

be different in tdTomato ${ }^{+}$and tdTomato ${ }^{-}$neurons. The same method was applied to rise times.

\section{Experiment design and statistical analyses}

Eight Gad2-tdTomato mice were used [7 males and 1 female (Mouse 2); 12-32 weeks old at the time of recording]. Imaging experiments were conducted in one bulb from each animal. The glomerulus-level analysis of onset latency (Fig. 6) includes data from 12 glomeruli in 7 mice. The glomerulus-level analysis of rise time (Fig. 8) includes data from 10 glomeruli in 6 mice. The comparisons between tdTomato ${ }^{+}$and tdTomato ${ }^{-}$neurons (Figs. 6, 8B) were conducted on fewer cells and glomeruli, those that satisfied additional criteria (see Results).

Normality of the data are not assumed in the statistical tests. $P$ values $<0.05$ were considered statistically significant. The following is a list of the statistical tests used; Figure 4D: Mann-Whitney $U$ test; Figure 5B: KruskalWallis test, for comparisons among cell-odor pairs; Figure 6: Kruskal-Wallis test for differences across glomerulus-odor pairs (in median onset latencies and in single trial onset latencies), and Mann-Whitney $U$ test for the comparison of glomerulus-controlled relative onset latency (see the previous section) between tdTomato ${ }^{+}$ and tdTomato ${ }^{-}$neurons; Figure 7C: Wilcoxon signed rank test; Figure 8B: Kruskal-Wallis test for differences across glomerulus-odor pairs, and Mann-Whitney $U$ test for the comparison of glomerulus-controlled relative rise time between tdTomato ${ }^{+}$and tdTomato ${ }^{-}$neurons; Figure $8 \mathrm{C}$ : Mann-Whitney $U$ test for the comparison of normalized rise times (in logarithm) between the different subsets of data (for details, see Fig. 8C, legend) with no correction for multiple comparisons.

\section{Results}

To analyze temporal coordination among JG cells in the glomerular network, we paid special attention on PG cells. PG cells are monoglomerular and are thus thought to receive little or no direct input from other glomeruli (Wachowiak and Shipley, 2006; Kiyokage et al., 2010). In addition, they are the most abundant cell type in the GL and can be genetically labeled. We thus thought that PG cells would be an excellent reference population in the analysis of possible functional heterogeneity among JG cells. Rather than selectively expressing the genetically encoded calcium indicator (GECl) GCaMP6f in PG cells, we genetically labeled $P G$ cells with a red-fluorescent protein tdTomato and expressed GCaMP6f via an injected AAV vector under the pan-neuronal synapsin promoter. This labeling strategy allowed us to record from genetically identified PG cells as well as neighboring neurons of other types.

\section{Expression pattern of fluorescent proteins}

We used GAD2 (GAD65) as a molecular marker for a subset of PG cells (Kiyokage et al., 2010) in the GL, and genetically labeled this subset with a cre-dependent tdTomato reporter mouse (Madisen et al., 2010) that was crossed with a Gad2-IRES-Cre mouse (Taniguchi et al., 2011). First, we examined the expression pattern of tdTomato (Fig. 1). tdTomato expression was observed in the GL and all inner layers of the OB. We occasionally observed off-target tdTomato expression in a small subset 

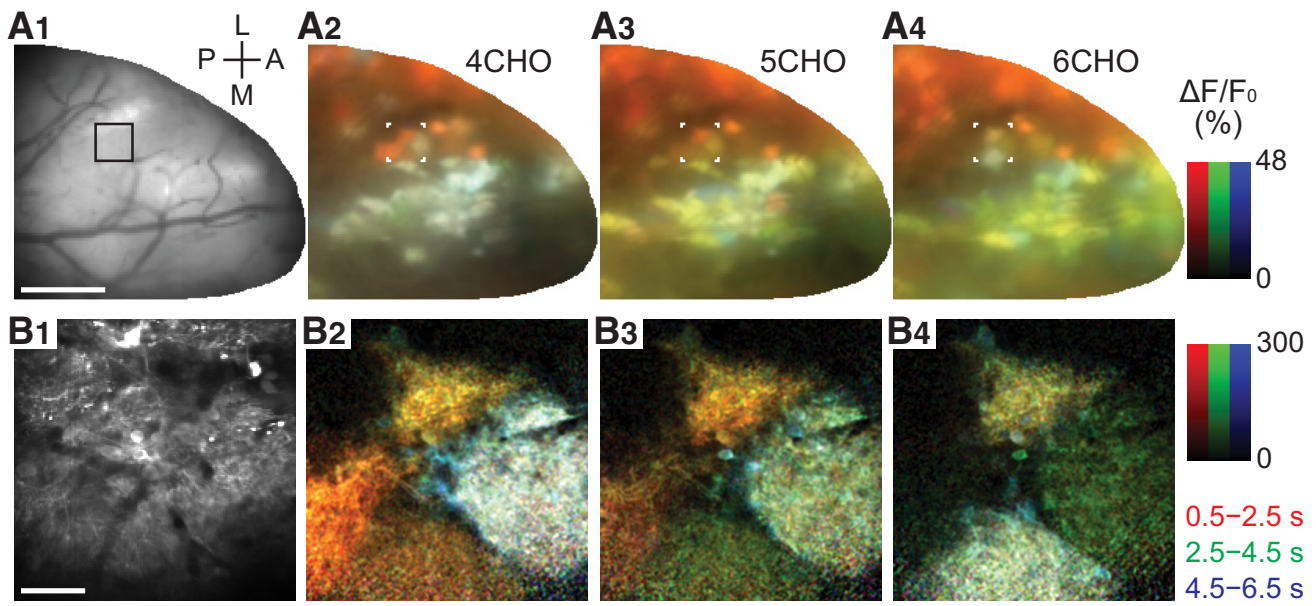

Figure 2. Activity $\left(\Delta F / F_{0}\right)$ maps color-coded by the phase of response in wide-field and two-photon imaging. A1, Surface image for wide-field (single-photon) imaging. L: lateral, A: anterior, M: medial, P: posterior. Scale bar, $500 \mu \mathrm{m}$. A2-A4, Maps of odor-evoked response to $4 \mathrm{CHO}, 5 \mathrm{CHO}$, and $6 \mathrm{CHO}$, respectively. Each map is a synthesis of red, green, and blue maps that represent the early $(0.5-2.5 \mathrm{~s})$, intermediate $(2.5-4.5 \mathrm{~s})$, and late (4.5-6.5 s) periods after stimulus onset, respectively. The duration of stimulus was $2 \mathrm{~s}$. This visualization allows us to map the heterogeneity of response time courses. For example, glomeruli that declined quickly after the stimulation appear in red (e.g., lateral glomeruli in A2-A4), whereas those with persistent response appear in yellow or white as they maintained the high-level calcium over the first two or all three periods (e.g., subsets of medial glomeruli in $\boldsymbol{A 2}-\boldsymbol{A 4}$ ). Glomeruli that appear in bluish colors are slow-rising ones. B1, A two-photon image of the selected area, indicated by the black box in $\boldsymbol{A 1}$ and by white corners in A2-A4. Scale bar, $50 \mu \mathrm{m}$. B2-B4, Maps of odor-evoked response, with the colors representing the same periods as in $\mathbf{A 2}-\boldsymbol{A 4}$.

of OSN axon bundles as well (data not shown). In the GL, while many JG-cell somata were labeled with tdTomato, fluorescence in the glomeruli was less prominent. Nearly all tdTomato ${ }^{+}$somata were also positive for GABA immunolabeling (Fig. 1A). A previous study reported that $\sim 37 \%$ of JG cells were positive for GAD65, $\sim 32 \%$ were positive for GAD67, and $\sim 16 \%$ were positive for both GAD67 and GAD65 (Parrish-Aungst et al., 2007). Therefore, we expected to observe a significant proportion of $\mathrm{GABA}^{+}$/ tdTomato $^{-}$cells, corresponding to $\mathrm{GAD}^{+} 7^{+} / \mathrm{GAD} 65^{-}$ cells, but this was not the case. We speculate that some of the GAD67 ${ }^{+} / \mathrm{GAD}^{-} 5^{-}$cells may have expressed Crerecombinase (under the Gad2 promoter) earlier in the animal's life and thus expressed tdTomato. In this scenario, tdTomato ${ }^{+}$neurons would represent both GAD65 ${ }^{+}$ JG cells and a subset of GAD $67^{+} /$GAD $65^{-}$cells. This raises the possibility that SSA cells, a separate class of JG cells positive for GAD67 (Kiyokage et al., 2010), were included in the tdTomato ${ }^{+}$cells. Therefore, we next examined the coexpression of tdTomato and tyrosine hydroxylase (TH), which is a marker of sSA cells in the OB (Kiyokage et al., 2010; Banerjee et al., 2015; Fig. 1B). We found that $\mathrm{TH}^{+}$cells included both tdTomato ${ }^{+}$and tdTomato ${ }^{-}$subpopulations (Fig. 1B, white and yellow arrowheads). This result is qualitatively consistent with the report that a subpopulation of $\mathrm{TH}^{+} \mathrm{JG}$ cells expresses GAD65 (Parrish-Aungst et al., 2007). In a previous study (Wachowiak et al., 2013), TH expression in JG cells was compared with GCaMP3 expression driven by the same mechanism as tdTomato in this study (a cre-dependent GCaMP3 reporter mouse crossed with the same Gad2IRES-Cre driver mouse). They reported that $14 \%$ of $\mathrm{TH}^{+}$ cells coexpressed GCaMP3, whereas the proportion of $\mathrm{TH}^{+} /$tdTomato $^{+}$cells among $\mathrm{TH}^{+}$cells in this study ap- pears higher, for unknown reasons. To summarize, tdTomato expression was specific to GABAergic neurons but may not be restricted to $\mathrm{GAD} 65^{+}$neurons; thus we cannot rule out the possibility that a small proportion of tdTomato ${ }^{+}$cells might represent SSA cells. Because we did not find conclusive difference between tdTomato ${ }^{+}$and tdTomato ${ }^{-}$cells in the following analyses, we did not further characterize the identity of tdTomato ${ }^{+}$cells.

\section{Odor-evoked calcium transients in GCaMP-labeled glomeruli}

At the beginning of the recording session for each mouse, we checked the positions of glomeruli that were responsive to our stimulus set by recording the odorevoked response in a large portion of the dorsal bulb with a high-speed CCD camera (Fig. 2A). In Figure 2A2-A4, each panel is a combination of three activity maps that represent three different time windows (see Materials and Methods). The early response (0.5-2.5 s after stimulus onset) is presented in red, the responses in subsequent time windows (2.5-4.5 and 4.5-6.5 s) are presented in green and blue, respectively, and the three maps are color-merged to produce the final image. Therefore, reddish colors imply dominance of the early response and whitish colors imply long-lasting responses, for example. Consistent with previous reports (Mori et al., 2006), strong activity was elicited by aliphatic aldehydes with different carbon chains. The central region of the dorsal $\mathrm{OB}$ in Figure $2 A$ shows many globular and spatially distinct centers of activity, which each primarily represent the activity of an individual glomerulus. These glomeruli show relatively prolonged activity even after the odor stimulation (white or yellow regions). By contrast, the lateral side of the bulb (top of the images) shows activity that declines 
quickly after stimulus offset (red regions). There are also glomeruli that appear bluish in color (Fig. 2A3), which represents a slow-rising calcium signal that peaked long after the stimulus offset. It is apparent that the time courses of odor-evoked calcium transients are diverse and depend on the particular glomerulus-odor pair. Of note, multiple cell types contributed to these signals from wide-field imaging because GCaMP6f was expressed under the control of the synapsin promoter. Therefore, the signals reflect not only the glomerular activity but also the activity of somata and other neuronal processes, including the interglomerular processes of sSA cells (Kiyokage et al., 2010) and processes in the inner layers.

\section{Odor-evoked calcium transients in GCaMP-labeled JG cells}

For two-photon recordings of individual JG cells, we chose the recording location guided by the data from wide-field imaging. Briefly, we first identified a candidate location where we found a cluster of several glomeruli, each of which responded to our stimulus set with discriminable odor selectivity. At the location, we first conducted conventional XYT scans to assess the odor-evoked response of glomeruli and JG cells (Fig. 2B). On the basis of the shape and "color" of the activity maps, we could reliably match the glomeruli in the two-photon imaging with those in the wide-field imaging (Fig. 2). We kept the recording depth relatively shallow $(45-98 \mu \mathrm{m}, 62.8 \pm 16.6$ $\mu \mathrm{m}$ mean $\pm \mathrm{SD}$, from the dura mater) so that the glomeruli were included in the field-of-view. Because we needed to determine the recording sites [regions-of-interest (ROIs)] for random-access scanning before the recording, we next picked 15 cells on the basis of the two-photon response maps (Fig. $2 B$ ) and the two-photon microscope images including those during odor-evoked responses (Figs. 3B, 4B; see Materials and Methods). Among 120 cells from 8 recordings, 79 cells $(66 \%)$ were identified as tdTomato $^{+}(9.9 \pm 2.6$ per recording, mean \pm SD). The tdTomato ${ }^{-}$recorded cells may include GAD65 ${ }^{-}$PG cells, sSA cells, and ET cells. ET cells, however, may be underrepresented, considering that the AAV vector we used (AAV2/1) preferentially infects PG and/or sSA cells (Adam et al., 2014) and that the somata of ET cells are located in the deeper part of the GL (Pinching and Powell, 1971; Macrides and Schneider, 1982; Hayar et al., 2004). As both GCaMP6f and tdTomato labeling were relatively dense, we had little morphologic information of the individual recorded cells such as whether the dendrites were monoglomerular or multiglomerular.

Examples of odor-evoked calcium transients are presented in Figure $3 \mathrm{~A}$. Because the random-access recording method records data only from a set of predetermined ROls (Figs. 3B, 4B), the obtained data are not a series of $X Y$ images but multiple traces from the ROls (optical multi-ROI recording). Conventionally, odor-evoked responses are measured by presenting odors for several seconds ( $2 \mathrm{~s}$ in this study) as shown in Figure $3 A$ (left). The responses to a $2 \mathrm{~s}$ stimulus exhibited some diversity in their time courses. Whereas previous electrophysiological experiments demonstrated that odor-evoked responses of PG cells tend to occur as bursts of action potentials that are locked to the respiration cycle (Tan et al., 2010; Homma et al., 2013; Livneh et al., 2014), we observed respiration-locked modulation of calcium signals in only a minor subset of traces (Fig. 4A). In the neurons of mouse visual cortex, GCaMP6f was capable of resolving two spikes with an interval of $50-75 \mathrm{~ms}$ as a stepwise increase of calcium signal or those with an interval of $>100$ ms more clearly (Chen et al., 2013). As the duration of a respiration cycle in this study ranged $300-500 \mathrm{~ms}(120-$ 200 cycle/min), the intervals between the bursts might be marginal for being resolved clearly when the respiration was relatively high and the bursts themselves also occupy a significant portion of the cycle. The typical time course of the odor-evoked calcium transients with $2 \mathrm{~s}$ stimulation was a monotonic rise followed by a monotonic decline, at various rates for each (Fig. $3 A$ ). However, the number of respiration cycles for the transients to reach the peak was heterogeneous. In some cases, the signal reached its peak amplitude during the first odor inhalation, whereas in other cases it took two or more inhalations to reach the peak. Although such heterogeneity in the response time course of JG cells is consistent with that observed in previous calcium-imaging studies (Wachowiak et al., 2013; Adam et al., 2014), this heterogeneity complicates the physiologic implications of information extracted from the calcium signal (e.g., the peak amplitude). To obtain simpler and more comprehensible time courses, we used briefer stimuli that were controlled to the duration of a single respiration cycle (see Materials and Methods). Figure $3 A$, right, shows examples of responses to such single-cycle stimuli. As expected, we observed morestereotyped time courses with these stimuli than with multicycle (2 s) stimuli.

\section{Response-based estimation of associated glomerulus}

JG cells that are associated with the same glomerulus show highly correlated stimulus selectivity (Tan et al., 2010; Kikuta et al., 2013) likely because they receive most of inputs from the glomerulus, and are thought to be located in the proximity of the associated glomerulus (Homma et al., 2013; Kikuta et al., 2013; Adam et al., 2014; Braubach et al., 2018). We thus reasoned that we could infer the association between the recorded cells and a particular glomerulus from their odor response and the location of the cell body. By comparing the odorevoked responses to different odors, we observed that some of the recorded cells indeed showed highly correlated response profiles including odor selectivity (Fig. $3 C$ ). To systematically compare the odor responses, we used the data from single-cycle stimulation trials and calculated the response amplitude, defined as the integral of each $\Delta F / F_{0}$ trace (i.e., the area under the trace). The five-element response vectors (Fig. $3 C$, middle) that represent the response amplitudes to the five odors were often sufficient for comparison. However, we also considered the 10-element difference vectors that were composed of the differences between every pair of response amplitudes (Fig. 3C, right); these were useful to distin- 
A

Multi-cycle stimulus (2 s)

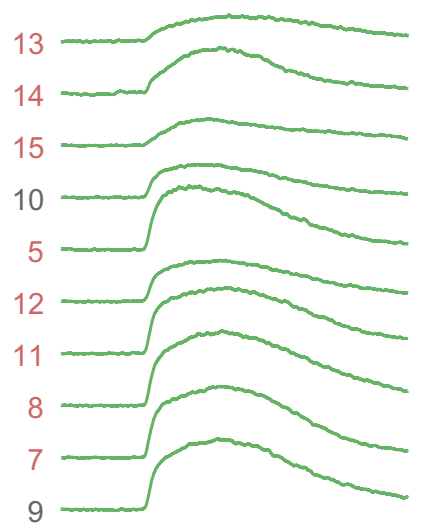

3 -

2

1

\section{Respiration}

Single-cycle stimulus
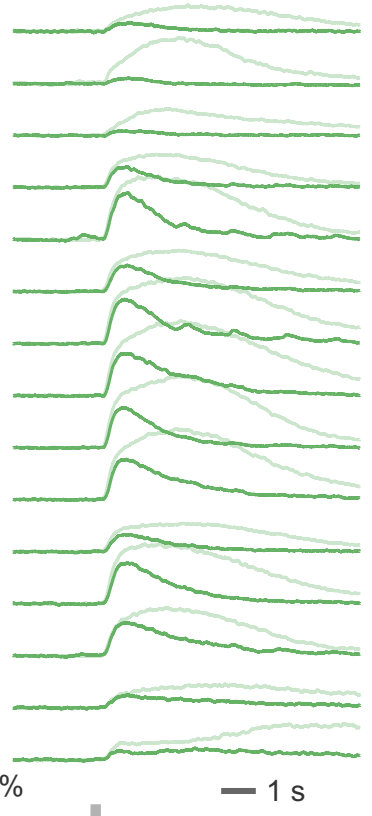

$\Delta \mathrm{F} / \mathrm{F}_{0} 100 \%$
$-1 \mathrm{~s}$
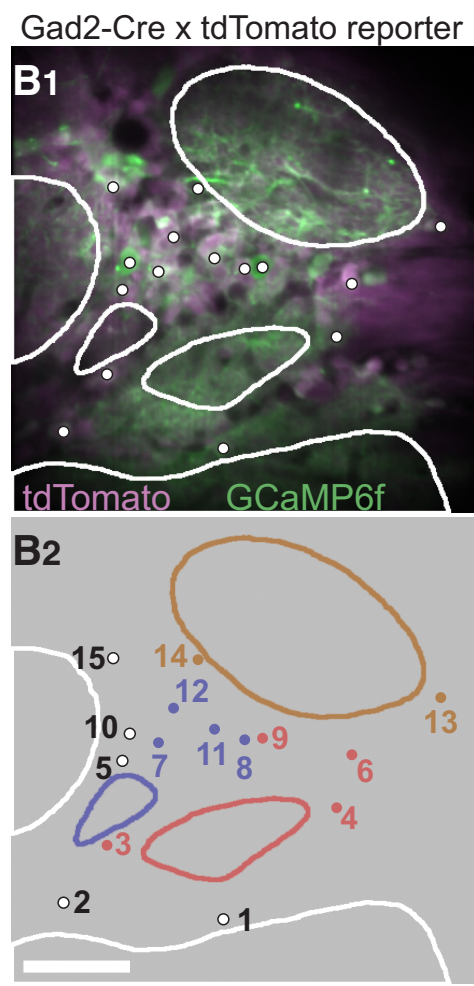

\section{C}

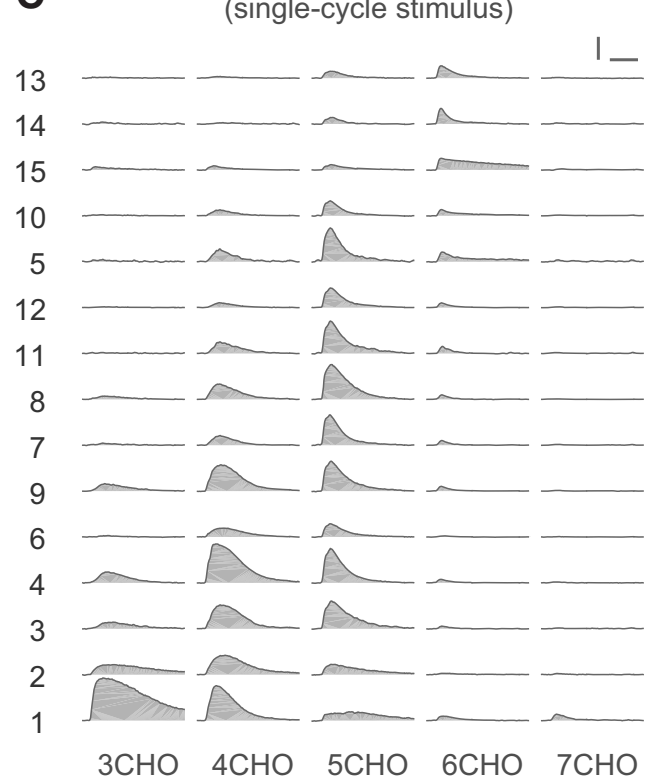

Time course

(single-cycle stimulus)

\section{Area under} trace (a.u.)

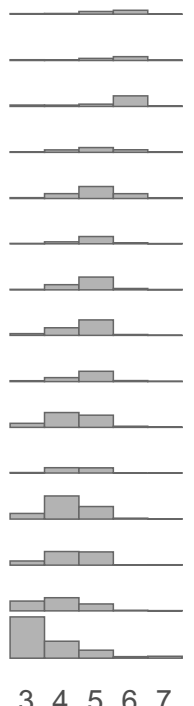

Difference between areas (a.u.)

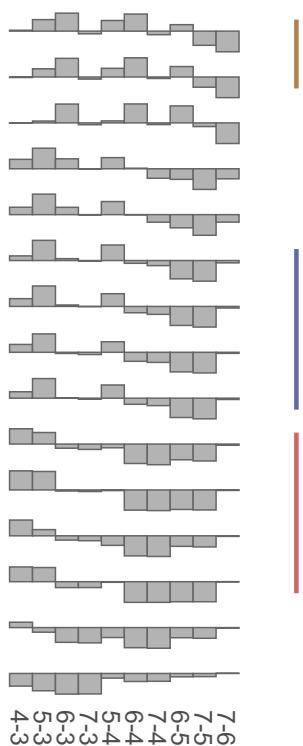

Figure 3. Odor-evoked calcium transients and grouping of JG cells by response profile. A, An example dataset from a single trial of random-access scanning of $15 \mathrm{ROls}$ (cells). Left traces are responses to a $2 \mathrm{~s}$ odor stimulus, and right traces are responses to a single-respiration-cycle stimulus. The bottom black traces show the respiration signal. Gray horizontal bars above the respiration signals indicate the timing of valve opening for odor presentation. Note that actual odor presentation lags $\sim 0.1 \mathrm{~s}$ behind the valve opening. The ordering of the ROls is intentional, based on the result of grouping shown in $\boldsymbol{C}$. $\boldsymbol{B}$, Two-photon image of the recording site (B1) and the ROI indexes (B2). To obtain the two-photon image of GCaMP6f (green), all XYT imaging data for all odors were averaged and then all poststimulus frames were averaged, solely for making the GCaMP fluorescence more visible. The tdTomato image (magenta) was obtained with a similar procedure, but an average of prestimulus frames was used. Dots and contours represent the ROIs and glomeruli, respectively. Non-white colors of dots and contours in $\mathbf{B 2}$ indicate the groups presented in $\boldsymbol{C}$. Scale bar, 50 $\mu \mathrm{m}$. C , Odor-evoked responses of the same ROls to five odors (single-cycle stimulus). The left block shows the response time courses. The middle block shows the areas under the time courses as bar charts. The right block shows the difference in areas between every possible pair of odors. Colored vertical lines at the right indicate the groups of cells putatively associated with the same 
continued

glomerulus (see text for details). Scale bars: horizontal, 3 s; vertical, $100 \% \Delta F / F_{0}$.

guish between the subsets of cells with relatively similar response vectors. Combining these data with the location of individual cells (Fig. $3 B$ ), we grouped together the cells that surrounded the same glomerulus and that showed highly correlated odor selectivity, and assumed that these neurons were associated with the same glomerulus (Fig. 3B2,C; see Materials and Methods). Note that only odor selectivity that was based on the time-averaged data (area under the trace) was used for this glomerulusassignment process (in addition to the location of cell
A

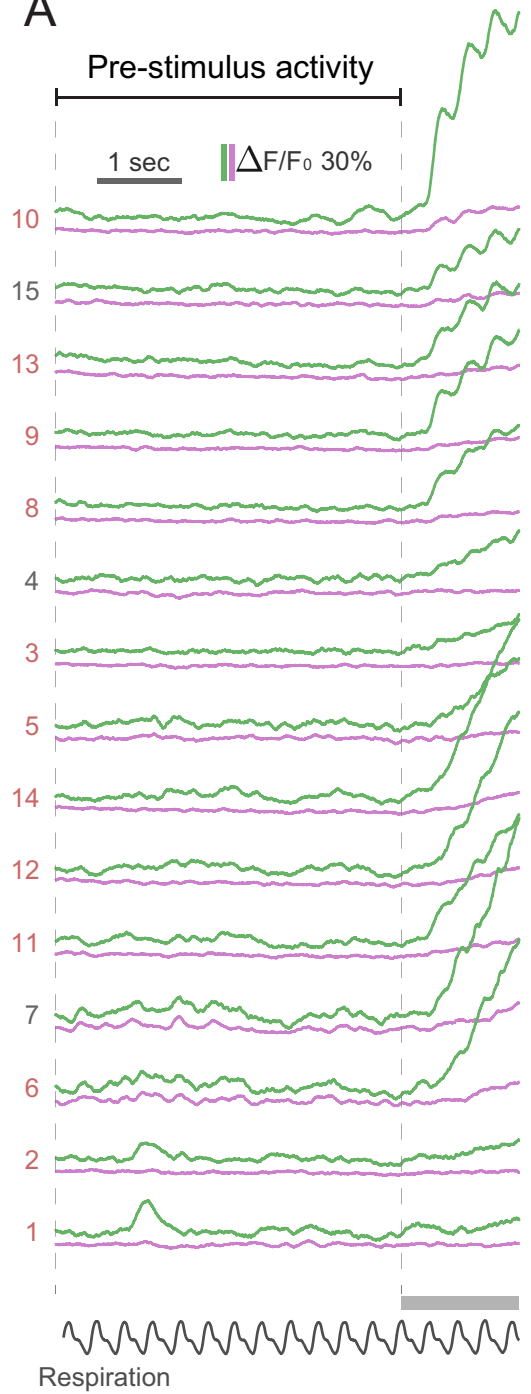

B Gad2-Cre $x$ tdTomato reporter

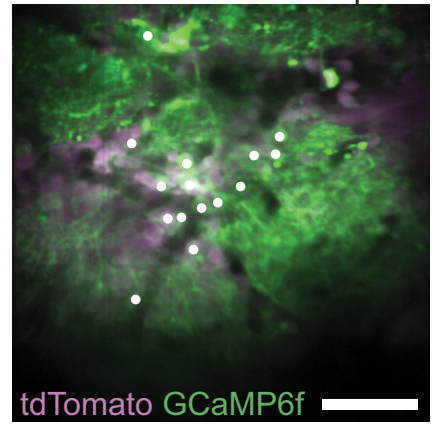

$15 \circ$

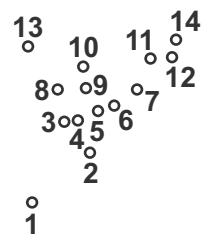

C
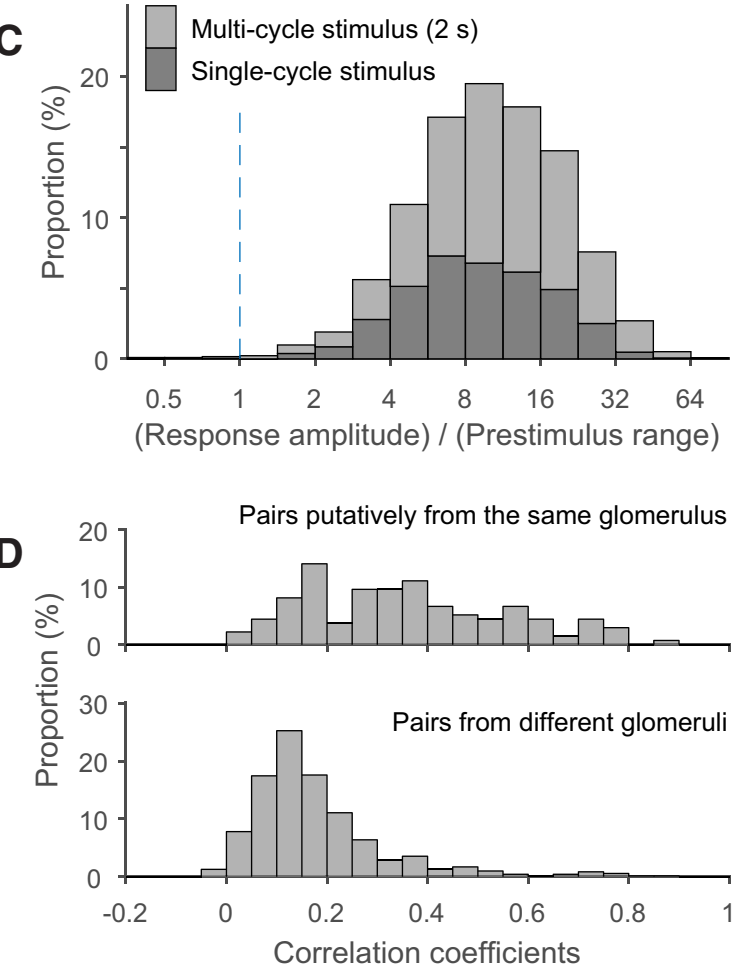

Figure 4. Characterization of calcium signals in the prestimulus period. $\boldsymbol{A}$, An example data of the prestimulus period and the initial part of odor-evoked response in a trial. Green traces are the signal from the green channel (GCaMP6f), and magenta traces are the signal from the red channel (tdTomato). Apparent odor-evoked changes in some of the magenta traces (e.g., ROI 10) suggest a minor contribution of the GCaMP signal to the red channel. The relative contribution from the GCaMP signal may vary depending on the relative expression levels of GCaMP and tdTomato at an individual ROI. Note clear respiration-coupled modulations of the odor-evoked response in the subset of traces. $\boldsymbol{B}$, Two-photon image of the recording site. The image was obtained with the same procedure for the image in Figure 3B1. ROls were indicated by white dots and the index of each dot is shown on the right of image. Scale bar, $50 \mu \mathrm{m}$. $\boldsymbol{C}$, Stacked histogram of the ratio between peak response amplitude and range (the distance between the 1st and 99th percentiles) in the prestimulus period. Note the logarithmic scale on the $x$-axis. The response is much larger than the fluctuations in the prestimulus period in the vast majority of cases. $\boldsymbol{D}$. Histograms showing the distribution of pairwise correlation coefficients for the time courses of activity during the prestimulus period. Top and bottom histograms show data from cell pairs putatively associated with the same glomerulus and pairs from different glomeruli, respectively. 
body). We deliberately avoided employing the temporal patterns of calcium transients during this process because we will discuss the dynamics of calcium transients with reference to these groups in the subsequent analyses. In the representative case shown in Figure 3, we identified two groups of four cells each (shown in red and blue). We also found another group with two cells (shown in brown), although this group was not considered in the subsequent analyses because it contained too few cells. We were able to find 14 groups of three or more cells that satisfied the criteria in the eight fields-of-view from eight mice.

\section{Baseline activity of the GCaMP-labeled JG cells}

Before a detailed analysis of the odor-evoked response, we briefly characterized the calcium signals in the prestimulus period and examined whether they could potentially affect the analyses of the odor-evoked responses (Fig. 4). Figure $4 A$ represents examples of traces during the prestimulus period. Whereas previous electrophysiological recordings reported that some JG cells exhibit action potentials that are coupled to the respiration cycle even without an odor stimulus (Homma et al., 2013, Livneh et al., 2014; Najac et al., 2015), we did not observe calcium signals that were regularly coupled to the respiration cycle in our cellular recordings despite a sufficiently high sampling rate. Possible explanations may include relatively few action potentials per cycle, relatively slow kinetics of either the GECI signal or intracellular calcium itself in the cell body, which may mask the respirationlocked modulation, or a combination of these. Alternatively, it may be also possible that we failed to reliably detect the individual cycle of stable respiration-locked bursts within the persistently elevated intracellular calcium that is caused by the burst themselves (Homma et al., 2013). In contrast to the (apparent) lack of respiration cycle-locked transients, we observed occasional fluctuations of the GCaMP signal that were absent or much less prominent in the simultaneously recorded red fluorescence from calcium-insensitive tdTomato. To compare the size of prestimulus fluctuations with the size of the odor-evoked response, we calculated the ratio of the peak amplitude of the odor-evoked response to the range (the difference between the 1st and 99th percentiles) of the prestimulus period signal (Fig. $4 \mathrm{C}$ ). In the vast majority of cell-odor pairs $(\mathrm{s} / \mathrm{n}>1$ in $99.7 \%$ of the cell-odor pairs with odor-evoked responses), the odor-evoked response was much larger than the prestimulus fluctuation, suggesting that the prestimulus activity would not significantly affect the analysis of odor-evoked calcium transients that we discuss in the following sections.

We also noticed that signal fluctuations during the prestimulus period tend to be synchronized among the cells putatively associated with the same glomerulus (see previous section). This observation suggests that prestimulus activity is driven by some form of glomerular input or spontaneous local network activity within a glomerulus. To examine this possibility, we calculated the pairwise Pearson's correlation coefficient between the time courses during the prestimulus period for all simultane- ously recorded pairs of cells. The distribution of correlation coefficients is presented separately for the cells putatively from the same glomerulus and all other pairs (Fig. 4D). The correlation coefficients were significantly higher in the "same glomerulus" pairs than in the "all others" pairs $\left(p=2.4 \times 10^{-25}\right.$, Mann-Whitney $U$ test for the same distribution, $N=135$ and 705 pairs of cell-odor pairs for the same glomerulus and all others groups, respectively). This result suggests some degree of synchrony in the prestimulus activity among the JG cells associated with the same glomerulus. There is a chance that the all other pairs may include some in which both cells were actually associated with the same glomerulus but that were mislabeled owing to our relatively conservative procedure for cell grouping.

\section{Glomerulus-specific onset latencies of odor-evoked calcium transients}

The somatic calcium transients primarily reflect the action potentials of the cell (Chen et al., 2013; Homma et al., 2013). Although it is difficult to infer the precise time course of bursts of action potentials from an odor-evoked calcium transient, it is more feasible to determine the onset latency of the transient, which reflects the onset of the burst. Therefore, we analyzed the onset latency of odor-evoked calcium (Figs. 5-7). First, we carefully determined the onset latency from our high-sampling-rate data (Fig. 5A; see Materials and Methods). Briefly, we approximate the earliest part of the rising signal as a linear line; the time of onset is defined as the time when this line intersects the baseline. The onset latency was defined as the difference between the time of onset and the onset of inhalation estimated from the respiration signal. Figure $5 B$ presents onset latencies from cell-odor pairs in which onset latencies were successfully determined in at least five trials. Although the trial-by-trial deviation was relatively large, as shown by the interquartile range (IQR) in Figure $5 B$, the median onset latency was not the same across cell-odor pairs $\left(p=1.8 \times 10^{-144}, N=165\right.$ cellodor pairs: Kruskal-Wallis test for non-uniform distribution). The median onset latencies of tdTomato ${ }^{+}$and tdTomato ${ }^{-}$cells were largely overlapping, with a trend that cell-odor pairs from tdTomato ${ }^{+}$cells had shorter onset latencies (Fig. 5C). In a simple statistical analysis in which all data points were treated as independent, this difference was statistically significant $(p=0.011$, MannWhitney $U$ test; tdTomato ${ }^{+}$: median $84 \mathrm{~ms}$, IQR $59 \mathrm{~ms}$, 105 cell-odor pairs; tdTomato ${ }^{-}$: median 108 ms, IQR 47 $\mathrm{ms}, 60$ cell-odor pairs). The distribution of onset latencies ranged from 0 to $150 \mathrm{~ms}$. Although we do not know precisely why the shortest onset latencies were so close to the onset of inhalation $(0 \mathrm{~ms})$, our speculation is that the respiration signals recorded from chest movements may lag behind the inhalation at the nose (Grimaud and Murthy, 2018). If this is indeed the case, then every onset latency reported in this study is underestimated by a certain amount.

The causes of heterogeneity in onset latency may include (1) heterogeneous inputs to different glomeruli, (2) differential interactions with the glomerular circuits, or (3) 
A

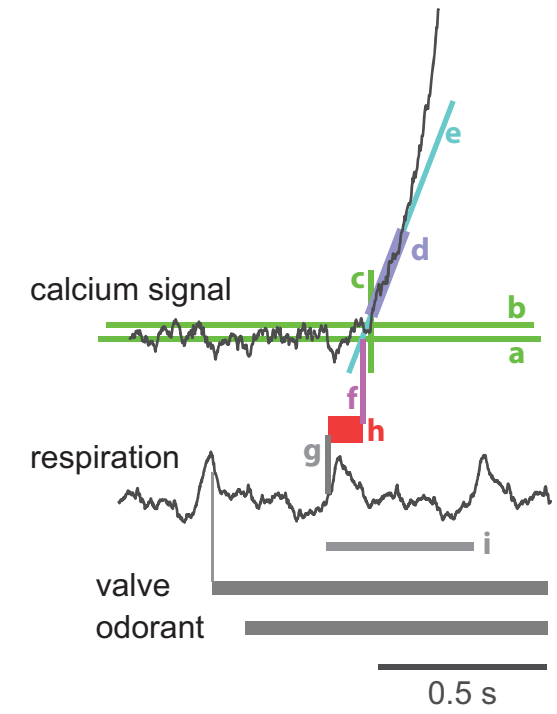

C

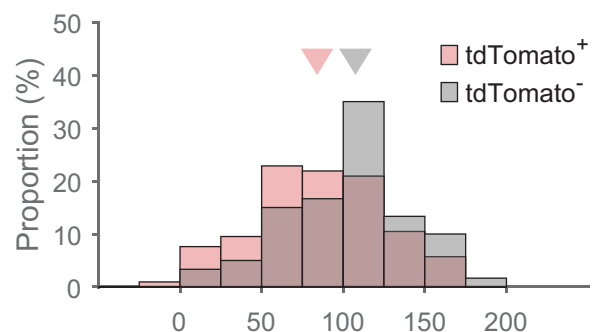

Onset latency from the inhalation onset (ms)
B

10

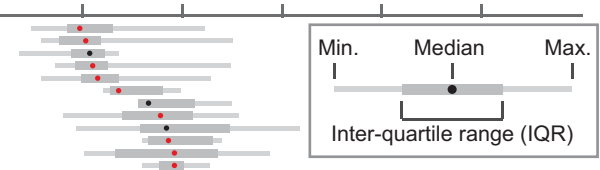

$20-$
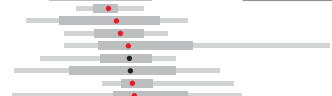

$-\frac{1}{2}$

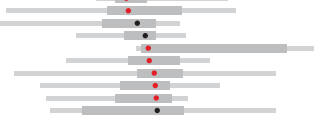

$30-$

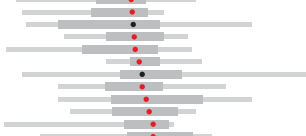

$40-$

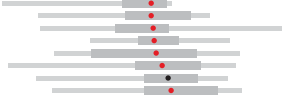

잉

$50-$

을

응 $60-$

凹

$70-$

$80-$

- tdTomato

$-$

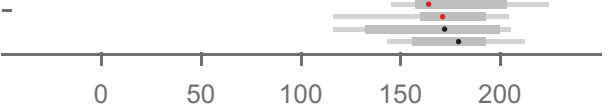

Onset latency from the inhalation onset (ms)

Figure 5. Onset latencies of odor-evoked calcium transients are heterogeneous across JG cells. $\boldsymbol{A}$, Graphic representation of the definition of onset latency. $\boldsymbol{A a}$, Baseline, defined as the mean of the prestimulus period signal. $\boldsymbol{A} \boldsymbol{b}$, Threshold, defined as 2.5 times the SD of the prestimulus period signal. Ac, Time point at which the signal exceeds the threshold. Ad, Time window of the first 100 $\mathrm{ms}$ above the threshold. $\boldsymbol{A e}$, Regression line of the signal in the $100 \mathrm{~ms}$ time window. Af, The point where the regression line crosses the baseline. This time point is considered the onset of the calcium transient. $\boldsymbol{A g}$, Onset of the first inhalation with the odor stimulus. $\boldsymbol{A} \boldsymbol{h}$, Onset latency, defined as the distance between the onset of inhalation and the onset of the calcium transient. Ai, One cycle of respiration. $\boldsymbol{B}$, Distribution of onset latencies. Each row corresponds to a single cell-odor pair and the distribution of onset latencies across repetitions is presented as a box-and-whisker plot (inset). Dots in the box-and-whisker plots represent the median, and expression of the GAD2 marker tdTomato is indicated by the dot color (red, tdTomato ${ }^{+}$; black, tdTomato ${ }^{-}$). Cell-odor pairs are arranged according to median onset latency and every other pair is presented for clarity (83 cell-odor pairs from 8 recording sites, out of all 165 pairs, are presented). Each box-and-whisker plot represents data from 5-17 trials. C, Overlapping histograms showing the distribution of medians shown in $\boldsymbol{B}$ for tdTomato $^{+}$(red) and tdTomato ${ }^{-}$(gray) cell populations. Triangles in the top indicates median for each population (tdTomato $^{+}: 84 \mathrm{~ms}, 105$ cell-odor pairs; tdTomato ${ }^{-}: 108 \mathrm{~ms}, 60$ cell-odor pairs; $p=0.012$, Mann-Whitney $U$ test).

differences in cell type. Because the majority of our recorded cells were PG cells, differences in cell type are unlikely to explain all of the heterogeneity. If heterogeneous inputs to different glomeruli are the primary cause, the deviation in onset latency should be much smaller among the JG cells associated with the same glomerulus. Therefore, we examined onset latencies in the groups of cell-odor pairs putatively associated with the same glomerulus to compare the onset latencies within and across glomeruli (Fig. 6). The median onset latencies of cell-odor pairs putatively associated with the same glomerulus were distributed in relatively narrow time windows compared with the distribution as a whole, suggesting that onset latency is primarily dependent on the glomerulus ( $p$
$=2.9 \times 10^{-9}, 88$ median onset latencies from 20 glomerulus-odor pairs, Kruskal-Wallis test for non-uniform distribution; Fig. 7C). Considering the relatively large IQRs across repetitions in each cell-odor pairs, we also applied the same statistical test for the onset latencies from individual trials (pooled over all cells in the given glomerulusodor pair) against glomerulus-odor pair, and obtained a consistent result $\left(p=3.5 \times 10^{-109}, 1003\right.$ single-trial onset latencies from 20 glomerulus-odor pairs, Kruskal-Wallis test). Importantly, there were cases in which different odors resulted in discriminable onset latencies for cells within the same glomerulus (Fig. 6, Mice 2 and 4), implying that the onset latency depends on both the glomerulus and the odor presented. This result also rules out the 


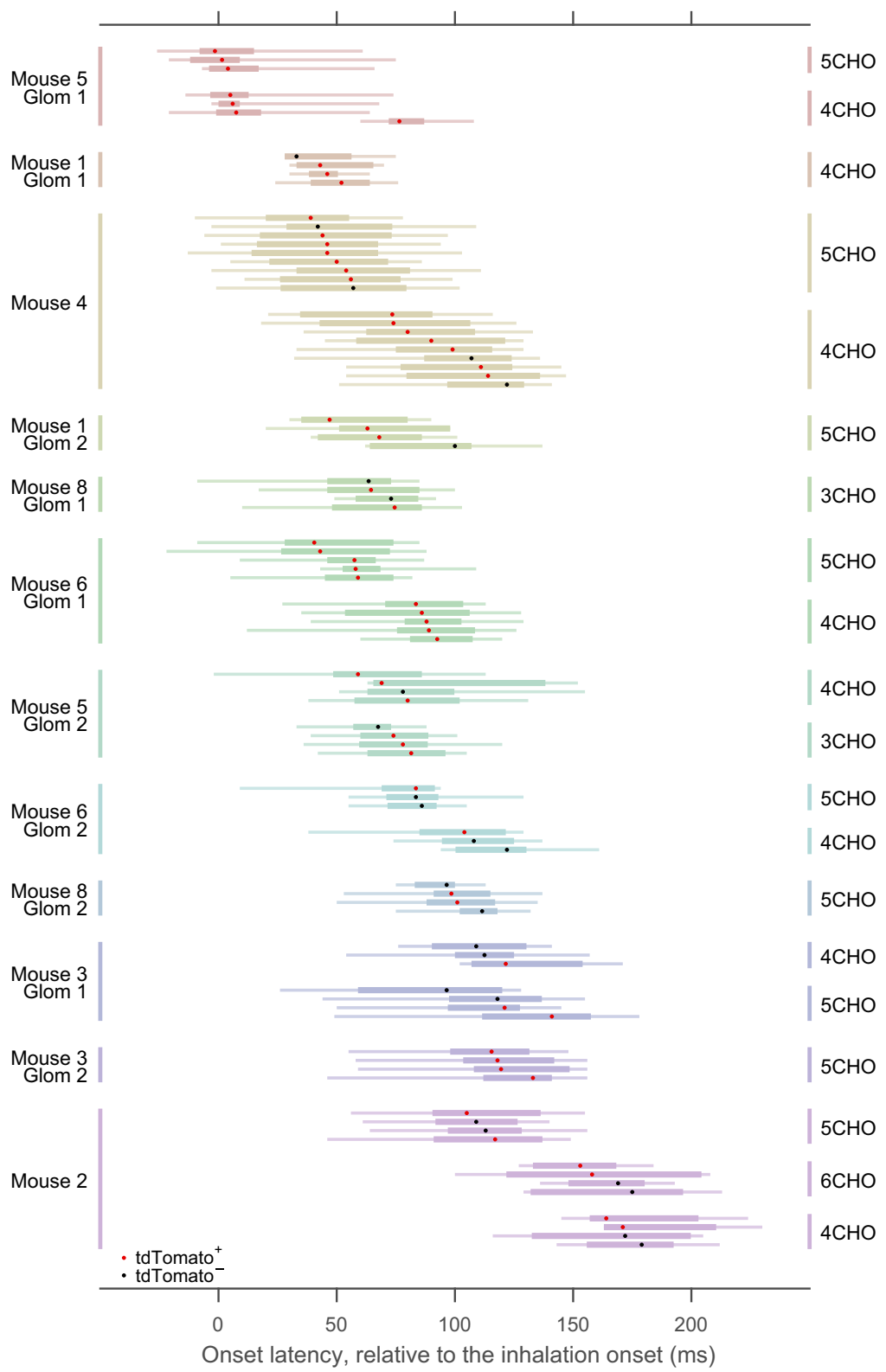

Figure 6. JG-cell onset latency strongly depends on the putative glomerular association. A subset of the box-and-whisker plot from Figure $5 B$ is presented, rearranged according to glomerulus-odor pairs. To clarify, cell-odor pairs in this figure include those not actually presented in Figure $5 B$, where only half of the cell-odor pairs are shown for clarity. As in Figure $5 B$, each row represents a single-odor pair, in which distribution of onset latency across repetitions was presented as box-and-whisker plots. Different glomeruli are presented in different colors. In some of the glomeruli, data from more than one odorant were available. Dots in the box-and-whisker plots represent the median, and expression of the GAD2 marker tdTomato is indicated by the dot color (red, tdTomato $^{+}$; black, tdTomato ${ }^{-}$). No statistical difference was observed between the onset latencies of tdTomato ${ }^{+}$and tdTomato $^{-}$ cells, considering the glomerulus-odor pairs (see text). Mouse and glomerulus identity are at the left. Odorant is indicated at the right.

possibility that the differences in onset latency are an artifact caused by the measurement error in inhalation onset.

Given that the glomerulus-odor pair was the primary determinant of onset latency, we re-examined the possible contribution of cell type to the onset latency by comparing tdTomato ${ }^{+}$and tdTomato $^{-}$cells in the same glomerulus-odor pairs. . We examined the relative order of onset latency within a glomerulus-odor pair (see Ma- terials and Methods) and did not see a difference with regard to tdTomato expression $(p=0.39, N=41$ tdTomato ${ }^{+}$and 26 tdTomato $^{-}$cell-odor pairs: Mann-Whitney $U$ test).

\section{Onset latencies of JG cells with the same odor- response profile}

To gain insight into the possible role of glomerular circuits in onset latency, we next compared the onset 
Onset latencies of 9 cells across repetitions (mouse 4)

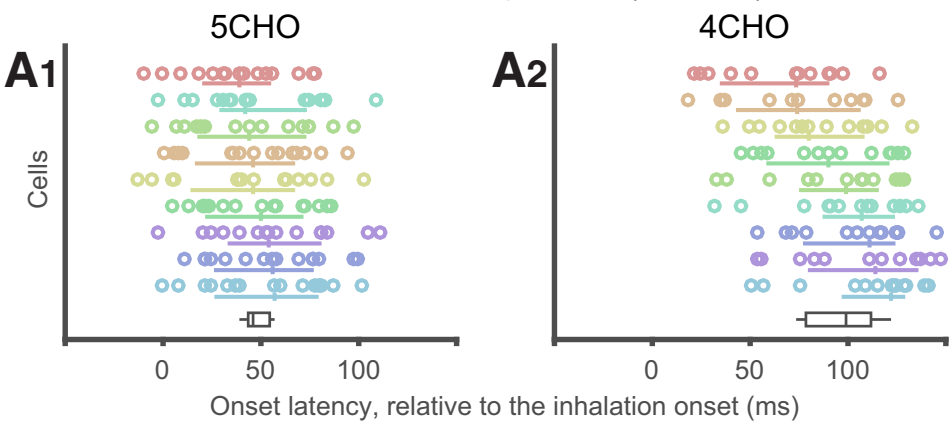

Onset latencies of the same cells in individual trials

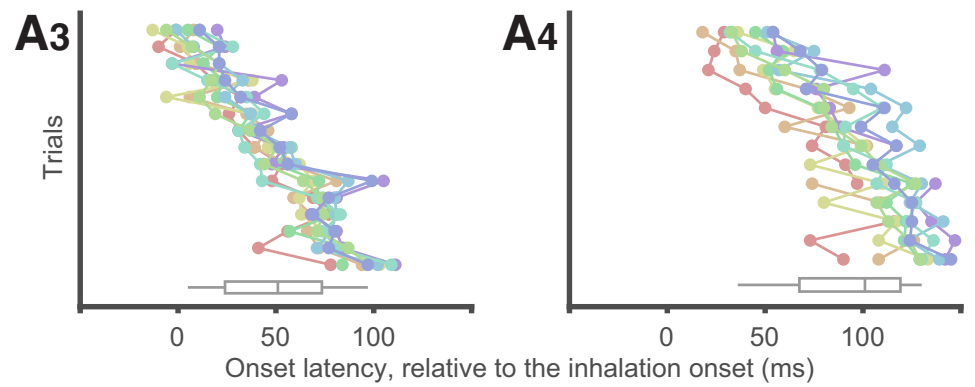

Onset latencies, corrected for the trial-by-trial deviation
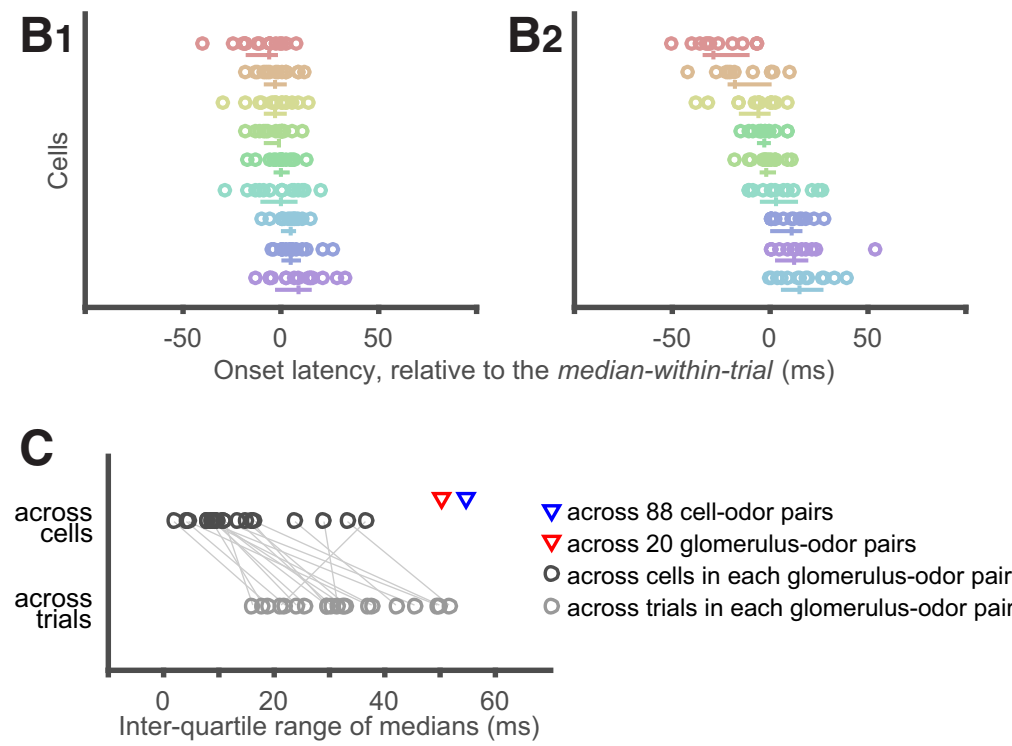

Figure 7. Detailed analysis of onset latency across cells putatively associated with the same glomerulus. The onset latencies of cell-odor pairs from a glomerulus in Mouse 4 (Fig. 6) are presented with an alternative visualization. A1, A2, Reconstruction of plots in Figure 6, except that each trial was explicitly plotted as circles. Each row represents an individual cell, as in Figure 6 . The median and IQR for each cell are presented as the accompanying vertical and horizontal lines, respectively. Colors represent individual cells and are preserved across all graphs in $\boldsymbol{A}$ and $\boldsymbol{B}$. The cells are sorted by their median onset latency. Left and right graphs show the responses to two different odors. Bottom, The black box-and-whisker plots show the distribution of median onset latency across cells. A3, A4, The same data as in $\boldsymbol{A 1}$ and $\boldsymbol{A 2}$, but rearranged so that each row represents an individual trial. Note that the variances within individual rows are markedly smaller than those in $\boldsymbol{A 1}$ and $\boldsymbol{A 2}$, suggesting that the primary cause of intra-cell deviation in $\boldsymbol{A 1}$ and $\boldsymbol{A} \mathbf{2}$ are trial-by-trial variability and not random errors. Trials are sorted according to their median onset latency, not by the order of acquisition. Bottom, The gray box-and-whisker plots show the distribution of median onset latency across trials. B1-A2, Onset latencies are plotted as in $\boldsymbol{A} \mathbf{1}$ and $\boldsymbol{A 2}$, except for that the contribution of trial-by-trial variance was removed. Specifically, the median onset latency across the in-group cells in the corresponding trial is subtracted from each data point. $\boldsymbol{C}$, IQRs of median onset latency across cells (black circles, corresponding to the black box-and-whisker plots in A1, A2) and across trials (gray circles, corresponding to the gray box-and-whisker plots in $\mathbf{A 3}, \mathbf{A 4}$ ) in each glomerulus-odor pairs are compared for all glomerulus-odor pairs shown in Figure 6. IQRs across cells are smaller in nearly all cases. Red triangle indicates the IQR of onset latency across all glomerulus-odor pairs involved in this analysis $(50 \mathrm{~ms}$ ). (The onset latency of a glomerulus-odor pair is defined as the median of medians across 
continued

involved cells.) This value is much larger than IQRs across cells in each glomerulus-odor pairs (black circles), but is close to the IQRs across all cell-odor pairs pooled (blue triangle, $55 \mathrm{~ms}, 88$ cell-odor pairs), suggesting that the spread of IQRs across all cell-odor pairs are primarily accounted for by the spread across glomerulus-odor pairs.

latency among cells within the same glomerulus-odor pair (Fig. 7). The first observation that drew our attention was that the trial-by-trial deviation of each cell (summarized as the $\mathrm{IQR}$ ) was larger than the deviation of median onset latencies across cells (Figs. 6, 7A1,A2). To understand the origin of this deviation, we reorganized the data and sorted them by trial (Fig. 7A3,A4). We found that the deviation of onset latencies within a trial (across cells) is much smaller than the deviation within a cell (across repetitions). In other words, the onset latencies were more deviated across trials (Fig. 7A3,A4, gray box-whisker plots) than across cells (Fig. $7 A 1, A 2$, black box-whisker plots), suggesting that the large intra-cell deviations are explained by the deviation across trials, which might be related, for example, to a fluctuating state in the animal or to measurement error in the inhalation onset. Of note, we did not find a clear relationship between the median onset latency within the trial and the order of acquisition (data not shown). As presented in Figure 7C, the IQR across trials was larger than the IQR across cells for most of the glomerulus-odor pairs ( $p=2.2 \times 10^{-4}, N=20$ : Wilcoxon's signed rank test). Therefore, when the onset latencies were aligned to the median of the corresponding trial, the deviation of each cell across trials substantially decreased (Fig. 7B). In the case presented in Figure 7B, the order of the aligned onset latencies was well preserved between the two stimuli (note that the same color represents the same cell). Although this may imply the presence of a systematic difference in onset latency among these cells, examining this further would require a larger dataset than we currently have, with multiple stimuli and more cells.

\section{Distribution of the rise time in single-cycle stimulation}

In addition to onset latency, an odor-evoked burst of action potentials can be characterized by, for example, the duration and the number of spikes. With the assumption that the duration of the rising phase of a calcium transient (rise time) is closely correlated with the duration of burst activity, we analyzed the rise time of odor-evoked calcium transients (Fig. 8). We defined the rise time as the duration for a calcium signal to rise from 20 to $80 \%$ of the peak amplitude. Because we could not distinguish individual respiration cycles in the calcium transients evoked by multi-cycle stimulation, we focused primarily on data from single-cycle stimulation.

First, we simply looked at the distribution of rise times across odor-cell pairs (Fig. $8 A$ ). As expected, the large majority $(84.8 \%)$ of rise times for single-cycle stimulation (blue) were shorter than $300 \mathrm{~ms}$, suggesting that the action potentials halted (or greatly decreased) within a single respiration cycle (300-500 ms). The presence of relatively short rise times may indicate spike activity of shorter duration in these cells. A minor proportion had longer rise times, likely reflecting the persistence of activity even after the stimulus offset. By contrast, the rise times $<2$ s stimulation (gray) were distributed more broadly, reflecting the heterogeneous time courses of calcium transients in this condition (Fig. $3 A$ ). Of note, as populations, rise time was not different between tdTomato $^{+}$and tdTomato $^{-}$neurons with both single-cycle stimulation (tdTomato $^{+}$: median $182 \mathrm{~ms}, N=108$ cellodor pairs; tdTomato ${ }^{-}$: median $165 \mathrm{~ms}, N=47$ pairs; $p=$ 0.25 , Mann-Whitney $U$ test) and $2 \mathrm{~s}$ stimulation (tdTomato $^{+}$: median $701 \mathrm{~ms}, N=213$ cell-odor pairs; tdTomato $^{-}$: median $596 \mathrm{~ms}, N=88$ pairs; $\left.p=0.093\right)$. Next, as for onset latency (Fig. 6), we grouped the data from cell-odor pairs putatively associated with the same glomerulus, to examine the contribution of glomerular inputs (Fig. 8B). With single-cycle stimulation, rise times depended on the glomerulus-odor pair with statistical significance $\left(p=1.2 \times 10^{-6}, 17\right.$ glomerulus-odor pairs, Kruskal-Wallis test for non-uniform distribution). This was not the case for the multi-cycle stimulation $(p=0.087)$. In Figure $8 B$, the IQRs for each cell-odor pair (horizontal bars) were not very different from the deviation among median rise times within the same group. This was in clear contrast with the results for onset latency (Figs. 6, 7A) and suggests little trial-by-trial deviation (see the previous two sections).

As for onset latency, above, we compared the relative rise times of tdTomato ${ }^{+}$and tdTomato $^{-}$neurons putatively associated with the same glomerulus and did not see any statistically significant differences $(p=0.28$ for single-cycle stimulation, $p=0.39$ for multi-cycle stimulation, $N=30$ tdTomato $^{+}$and 14 tdTomato $^{-}$cell-odor pairs from eight glomerulus-odor pairs that included four or more neurons: Mann-Whitney $U$ test). Finally, we examined the correlation between transient rise times and peak amplitudes, as a strong correlation between these would not be compatible with our assumption that rise time reflects the duration of burst activity. We only considered data from single-cycle stimulation in this analysis. Because we cannot compare response amplitudes across different cells, we analyzed cells that were activated by more than one odorant. For each cell, all rise times and response amplitudes were normalized to those of the response with the largest peak amplitude (the reference response), and plotted in Figure $8 C$ [reference responses are omitted but would appear at $(0,1)$ on the plot]. Among 77 cell-odor pairs, the rise time was shorter in 34 cellodor pairs and longer in 43 cell-odor pairs than the corresponding reference response. Thus, it is highly unlikely that rise time is accounted for solely by the response amplitude, which favors our assumption that rise time is correlated with the duration of burst activity. When we considered tdTomato ${ }^{+}$and tdTomato $^{-}$cells separately, both cell populations have rise time shorter than and longer than the reference response (tdTomato ${ }^{+}: 23$ 


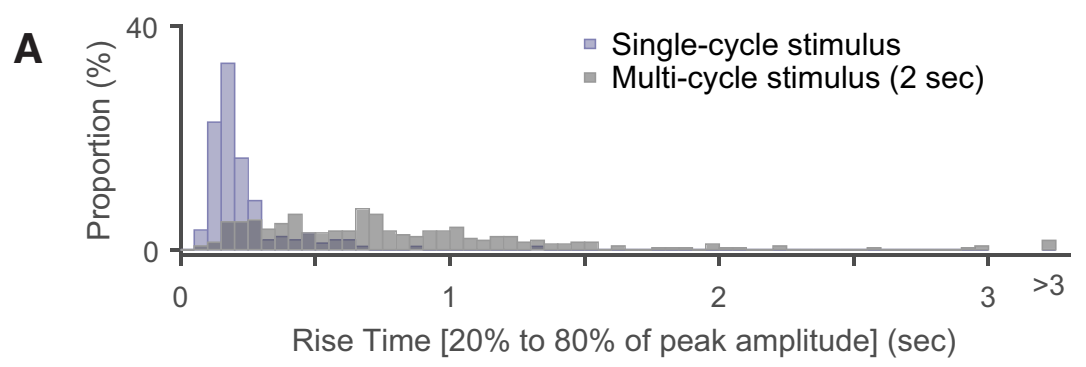

B Single-cycle stimulus Multi-cycle stimulus (2 s)
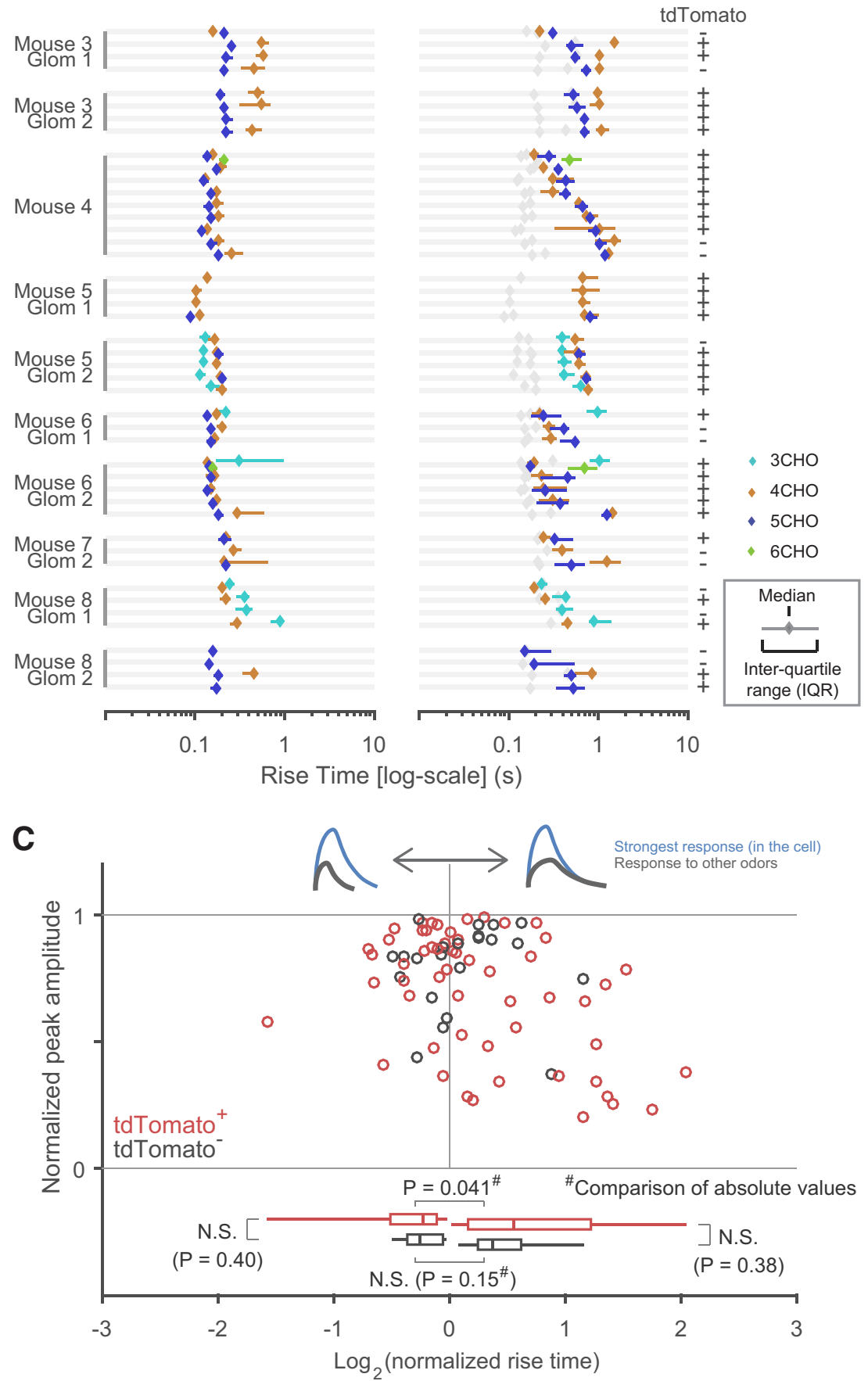

Figure 8. Analyses of the rise time of odor-evoked calcium transients. $\boldsymbol{A}$, Distribution of rise times, defined as the duration between the points when a signal reached 20 and $80 \%$ of peak amplitude. Two overlapping histograms are presented. The blue histogram 
continued

represents the distribution of single-cycle stimulation, and the gray one represents the distribution of multi-cycle (2 s) stimulation. $\boldsymbol{B}$, Rise times are presented for cells putatively associated with the same glomerulus. All cases in which rise time was successfully determined in three or more cells are presented. The median is presented as a diamond, and the interquartile range as a horizontal bar. No horizontal bar means that the interquartile range is smaller than the size of the diamond marker. Right (multi-cycle stimulation), the data from the left (single-cycle stimulation) are replicated in gray to facilitate comparison. Note the logarithmic scale on the $x$-axis. Expression of the GAD2 marker tdTomato [positive (+) or negative (-)] is indicated at the right. The indexes for mouse and glomerulus are shown at the left: these indexes correspond to those in Figure 6. $\boldsymbol{C}$, The relationship between onset latency and peak response amplitude is plotted. Because peak amplitudes can be compared only within the same cell, only data from cells in which the rise time was successfully determined for more than one odorant were used in the analysis (see text). Red and black circles represent the data point from tdTomato ${ }^{+}$and tdTomato $^{-}$cells, respectively. Bottom, The set of box-and-whisker plots show the distribution of tdTomato $^{+}$and tdTomato ${ }^{-}$cells, in which cell-odor pairs with shorter rise time than the reference response and those with longer rise time are considered separately. The difference of distribution is statistically tested for matching pairs, where negative normalized rise times in logarithm are converted to their absolute values (tdTomato ${ }^{+}: 32$ cell-odor pairs with normalized rise time $>1,22$ pairs with $<1$; tdTomato $^{-}: 11$ cell-odor pairs with normalized rise time $>1,11$ pairs with $<1$, Mann-Whitney $U$ test). Note the logarithmic scale on the $x$-axis.

shorter and 32 longer cell-odor pairs; tdTomato ${ }^{-}: 11$ shorter and 11 longer cell-odor pairs). And the distribution of normalized rise times were overlapping between tdTomato ${ }^{+}$and tdTomato ${ }^{-}$cells, except that the cellodor pairs with the largest normalized rise times $\left[\log _{2}(\right.$ normalized rise time) $>1]$ were mostly associated with tdTomato ${ }^{+}$(Fig. 8C). This subpopulation might represent a distinct subtype of PG cells.

\section{Discussion}

In this study, we recorded odor-evoked calcium transients from individual JG cells in anesthetized freebreathing mice at an extremely high sampling rate and measured the onset latency and rise time of the transients. We examined these properties in sets of JG cells putatively associated with the same glomerulus ("homoglomerular" JG cells). Although the onset latency of JG cells spans $\sim 150 \mathrm{~ms}$ when glomerular association is not taken into account, the range of onset latencies is substantially smaller (typically a few tens of milliseconds) among homoglomerular JG cells. Similar glomerulus specificity was also found for rise times when we presented a brief odor stimulus. These observations suggest that the glomeruli (more strictly, the glomerulus-odor pairs) determine a significant portion of the response time course in JG cells.

\section{High-speed calcium recording by random-access scanning}

Here, we briefly discuss the technical aspects of this study. The intracellular calcium level has become a popular proxy for neuronal activity (Grienberger and Konnerth, 2012). It is well acknowledged that the calcium signal is much slower than the related action potentials, but this has not been a serious issue in two-photon imaging studies partly owing to the low frame rates of conventional two-photon microscopes. At the same time, such a situation has limited the analyses possible from readouts of the temporal patterns of calcium signals in vivo. In this study, we used a random-access scanning technique (lyer et al., 2006; Grewe et al., 2010) and sampled the calcium transients at a high rate $(667 \mathrm{~Hz})$. This high sampling rate was achieved by limiting the number of pixels sampled. Thus, the sampling duration per pixel, which often limits the $s / n$ ratio of two-photon recording, was no different from the full-frame scan. Although randomaccess scanning has tended to be used more for volumetric recordings of numerous cells (Göbel et al., 2007; Froudarakis et al., 2014), high-fidelity recording is another powerful application of the technique (Grewe et al., 2010). Technically, our scanner is capable of scanning at perpixel sampling rates up to $\sim 10$ times higher than in this study (with lower $s / n$ ratio). Thus, this technique is compatible with two-photon voltage recording of action potentials with voltage-sensitive fluorescence proteins, which have been continuously improving in efficacy (Lin and Schnitzer, 2016; Chamberland et al., 2017).

\section{Functional feature of GAD2 positive and negative cells}

Against our expectations, we did not find a clearly distinct functional difference between GAD2-positive (tdTomato $^{+}$) and GAD2-negative cells (tdTomato ${ }^{-}$) in this series of experiments. Although tdTomato ${ }^{+}$cells had a shorter onset latency than tdTomato ${ }^{-}$cells in a simpler analysis (Fig. 5), our dataset was not sufficiently large for clarifying whether this shorter onset latency is truly associated with the tdTomato expression or caused by other reasons such as uneven sampling of tdTomato ${ }^{+}$and tdTomato ${ }^{-}$cells across glomeruli. In addition, we did not see the difference in the rise time either (but see Fig. $8 C$ ). We thus did not reach a firm conclusion about the difference in two populations, at the temporal resolution of our methods. Of note, tdTomato ${ }^{-}$cells were very likely composed of multiple cell types and tdTomato ${ }^{+}$cells could also include multiple subtypes of PG cells (Shao et al., 2009; Najac et al., 2015). Heterogeneity within the tdTomato $^{+}$or tdTomato ${ }^{-}$cells may have had a negative impact in our analyses.

\section{Heterogeneity in the dynamics of odor-evoked calcium transients}

In experiments with anesthetized free-breathing animals, it is common practice to present an odor stimulus for several seconds, lasting over multiple respiration cycles. In our recording of odor-evoked calcium transients in the GCaMP6f-expressing JG cells (dominated by PG cells), it took a different number of respiration cycles to 
reach the transient peak in different cells (or cell-odor pairs); this is also illustrated by the heterogeneity in rise times (the period from 20 to $80 \%$ of the peak signal amplitude; Fig. $8 A, B$ ). It is not clear to what extent this heterogeneity is due to the spike-calcium relationship (Grienberger and Konnerth, 2012) or to the temporal pattern of the spikes. The phenomenon may potentially be understood better by inferring the action potentials from the calcium signals (Yaksi and Friedrich, 2006; Theis et al., 2016; Rahmati et al., 2018). In this study, we did not take this path because of our lack of easy means to validate the inference. It is indeed an interesting question whether such methods could successfully reconstruct the action potentials in this case: reconstruction may be challenging in PG cells owing to the respiration-coupled bursting activity that may make the spike-calcium relationship strongly non-linear.

Such heterogeneous time courses also raise a practical problem for quantifying the amplitude of calcium transients. For example, peak amplitude is often used to quantify the amplitude of transients, but it may be associated with different numbers of respiration cycles across cells. Note that this issue is also relevant to the analysis of rise time. In this study, we used single-respiration-cycle stimuli to prevent signals from accumulating over multiple respiration cycles for analyses involving comparisons of the response amplitude (Figs. 3, 8C). Although we do not have enough data to address whether the problem is also relevant to other types of neurons in the olfactory system, diverse calcium-transient time courses have been reported in multiple cell types (Wachowiak et al., 2013). Thus, there may be other cases where the shape of the calcium transients deserves consideration during the quantification of response properties.

\section{Glomerular circuits and glomerulus-specific onset latency}

The glomerulus-specific onset latencies of JG cells are characterized by relatively small deviations in onset latency within the same glomerulus and larger deviations across glomeruli. The most plausible explanation for this is that the JG cells follow the onset of sensory inputs in each glomerulus, as the onset latency of sensory inputs from OSNs is heterogeneous across glomeruli (Spors et al., 2006; Carey et al., 2009). On the other hand, the small deviations within a glomerulus may imply that the sensory inputs from many OSNs are accumulated in such a time window, given that an axon from each OSN covers a small fraction of a glomerulus (Hálasz and Greer, 1993). It may then be reasonable that the recorded JG cells, regardless of tdTomato expression, are activated within a time window of $\sim 20 \mathrm{~ms}$ (Fig. 7), because most (if not all) JG cells receive the inputs from OSNs directly or disynaptically via the local neurons (Wachowiak and Shipley, 2006; Tavakoli et al., 2018). As discussed above, tdTomato ${ }^{+}$and tdTomato ${ }^{-}$cells would be driven either in parallel or with a finer difference than what was considered in this study. In either case, such a temporally confined activity of excitatory and inhibitory neurons could play important roles in relaying the dynamic inputs from the OSNs to the principal neurons. It is worth noting that most of our analyses were limited to relatively strong responses because we only used data with a high $\mathrm{s} / \mathrm{n}$ ratio. It is thus an interesting question whether a weaker stimulus would broaden the distribution of onset latencies among homoglomerular JG cells. If this was indeed the case, such a mechanism could play an important role in the intraglomerular circuit, where many JG cells of the same type (or subtype) receive homogeneous inputs and perform the same function (e.g., feedforward excitation/ inhibition). For instance, even if a class of JG cells collectively releases the same total amount of neurotransmitter to the glomerular circuit, the consequences would be different if highly synchronized neurons release the transmitter at a high density over a short time window, versus if less-synchronized neurons release the transmitter at a lower density over a longer time window. We suggest such a mechanism may be useful to enhance the $\mathrm{s} / \mathrm{n}$ ratio of sensory inputs to the glomerulus while maintaining their dynamics.

\section{Implications for the function of the $\mathrm{OB}$ and glomeruli}

Although our recordings were limited to JG cells, the glomerulus-specific response time course of these cells suggests that the time course of sensory inputs is highly glomerulus-specific. Each glomerulus was driven at a different time, with a strong input in the first tens of milliseconds. The temporal pattern of odor-evoked activity has been shown to be a part of stimulus representation in the principal neurons (Cury and Uchida, 2010; Uchida et al., 2014). Our results support the idea that sensory inputs play a role in the temporal coding of odor stimuli (Schaefer and Margrie, 2007; Raman et al., 2010). For example, a coarse temporal pattern may be determined by the sensory inputs (as well as the glomerular circuits) and be largely homogeneous among homoglomerular mitral cells. The odor-evoked activity, including the temporal pattern, may then be refined in subsequent $O B$ circuits so that it is diversified among individual principal neurons (Dhawale et al., 2010; Kikuta et al., 2013; Arneodo et al., 2018). Furthermore, glomerulus-specific onset latencies may play a role in the horizontal connections among glomeruli (Aungst et al., 2003; Kiyokage et al., 2010; Banerjee et al., 2015). Early responding glomeruli may be capable of modulating the activity in neighboring glomeruli more strongly, although the computational advantage of such a mechanism is not obvious in a system that supposedly lacks a topographic representation of stimulus space (Cleland and Sethupathy, 2006; Cleland, 2014). Similarly, lateral interactions between mitral/tufted cells may also be orchestrated by the differences in their onset latencies (Arneodo et al., 2018). At last, if these mechanisms make the onset latency (and may be the duration) of outputs from individual glomeruli more distinct, it would benefit the lateral interaction in the OB circuits as well as the integration of signals in the olfactory centers by limiting the number of glomeruli that can send the coincident inputs to the downstream neurons in a given moment. 


\section{References}

Adam Y, Livneh Y, Miyamichi K, Groysman M, Luo L, Mizrahi A (2014) Functional transformations of odor inputs in the mouse olfactory bulb. Front Neural Circuits 8:129. CrossRef Medline

Arneodo EM, Penikis KB, Rabinowitz N, Licata A, Cichy A, Zhang J, Bozza T, Rinberg D (2018) Stimulus dependent diversity and stereotypy in the output of an olfactory functional unit. Nat Commun 9:1347. CrossRef Medline

Aungst JL, Heyward PM, Puche AC, Karnup SV, Hayar A, Szabo G, Shipley MT (2003) Centre-surround inhibition among olfactory bulb glomeruli. Nature 426:623-629. CrossRef Medline

Banerjee A, Marbach F, Anselmi F, Koh MS, Davis MB, Garcia da Silva P, Delevich K, Oyibo HK, Gupta P, Li B, Albeanu DF (2015) An interglomerular circuit gates glomerular output and implements gain control in the mouse olfactory bulb. Neuron 87:193-207. CrossRef Medline

Braubach O, Tombaz T, Geiller T, Homma R, Bozza T, Cohen LB, Choi $Y$ (2018) Sparsened neuronal activity in an optogenetically activated olfactory glomerulus. Sci Rep 8:14955. CrossRef Medline

Burton SD (2017) Inhibitory circuits of the mammalian main olfactory bulb. J Neurophysiol 118:2034-2051. CrossRef Medline

Carey RM, Sherwood WE, Shipley MT, Borisyuk A, Wachowiak M (2015) Role of intraglomerular circuits in shaping temporally structured responses to naturalistic inhalation-driven sensory input to the olfactory bulb. J Neurophysiol 113:3112-3129. CrossRef Medline

Carey RM, Verhagen JV, Wesson DW, Pírez N, Wachowiak M (2009) Temporal structure of receptor neuron input to the olfactory bulb imaged in behaving rats. J Neurophysiol 101:1073-1088. CrossRef Medline

Chamberland S, Yang HH, Pan MM, Evans SW, Guan S, Chavarha M, Yang Y, Salesse C, Wu H, Wu JC, Clandinin TR, Toth K, Lin MZ, St-Pierre F (2017) Fast two-photon imaging of subcellular voltage dynamics in neuronal tissue with genetically encoded indicators. eLife 6:e25690. CrossRef Medline

Chen TW, Wardill TJ, Sun Y, Pulver SR, Renninger SL, Baohan A, Schreiter ER, Kerr RA, Orger MB, Jayaraman V, Looger LL, Svoboda K, Kim DS (2013) Ultrasensitive fluorescent proteins for imaging neuronal activity. Nature 499:295-300. CrossRef Medline

Cleland TA (2014) Construction of odor representations by olfactory bulb microcircuits. Prog Brain Res 208:177-203. CrossRef Medline

Cleland T, Sethupathy P (2006) Non-topographical contrast enhancement in the olfactory bulb. BMC Neurosci 7:7. CrossRef Medline

Cury KM, Uchida N (2010) Robust odor coding via inhalationcoupled transient activity in the mammalian olfactory bulb. Neuron 68:570-585. CrossRef Medline

De Saint Jan D, Hirnet D, Westbrook GL, Charpak S (2009) External tufted cells drive the output of olfactory bulb glomeruli. J Neurosci 29:2043-2052. CrossRef Medline

Dhawale AK, Hagiwara A, Bhalla US, Murthy VN, Albeanu DF (2010) Non-redundant odor coding by sister mitral cells revealed by light addressable glomeruli in the mouse. Nat Neurosci 13:1404-1412. CrossRef Medline

Froudarakis E, Berens P, Ecker AS, Cotton RJ, Sinz FH, Yatsenko D, Saggau P, Bethge M, Tolias AS (2014) Population code in mouse V1 facilitates readout of natural scenes through increased sparseness. Nat Neurosci 17:851-857. CrossRef Medline

Fukunaga I, Herb JT, Kollo M, Boyden ES, Schaefer AT (2014) Independent control of gamma and theta activity by distinct interneuron networks in the olfactory bulb. Nat Neurosci 17:12081216. CrossRef Medline

Geramita M, Urban NN (2017) Differences in glomerular-layermediated feedforward inhibition onto mitral and tufted cells lead to distinct modes of intensity coding. J Neurosci 37:1428-1438. CrossRef Medline
Göbel W, Kampa BM, Helmchen F (2007) Imaging cellular network dynamics in three dimensions using fast 3D laser scanning. Nat Methods 4:73-79. CrossRef Medline

Grewe BF, Langer D, Kasper H, Kampa BM, Helmchen F (2010) High-speed in vivo calcium imaging reveals neuronal network activity with near-millisecond precision. Nat Methods 7:399-405. CrossRef Medline

Grienberger C, Konnerth A (2012) Imaging calcium in neurons. Neuron 73:862-885. CrossRef Medline

Grimaud J, Murthy VN (2018) How to monitor breathing in laboratory rodents: a review of the current methods. J Neurophysiol 120:624632. CrossRef Medline

Hálasz N, Greer CA (1993) Terminal arborizations of olfactory nerve fibers in the glomeruli of the olfactory bulb. J Comp Neur 337:307316. CrossRef Medline

Hayar A, Karnup S, Shipley MT, Ennis M (2004) Olfactory bulb glomeruli: external tufted cells intrinsically burst at theta frequency and are entrained by patterned olfactory input. J Neurosci 24: 1190-1199. CrossRef Medline

Homma R, Kovalchuk Y, Konnerth A, Cohen LB, Garaschuk O (2013) In vivo functional properties of juxtaglomerular neurons in the mouse olfactory bulb. Front Neural Circuits 7:23. CrossRef Medline

Iyer V, Hoogland TM, Saggau P (2006) Fast functional imaging of single neurons using random-access multiphoton (RAMP) microscopy. J Neurophysiol 95:535-545. CrossRef Medline

Kikuta S, Fletcher ML, Homma R, Yamasoba T, Nagayama S (2013) Odorant response properties of individual neurons in an olfactory glomerular module. Neuron 77:1122-1135. CrossRef Medline

Kiyokage E, Pan YZ, Shao Z, Kobayashi K, Szabo G, Yanagawa Y, Obata K, Okano H, Toida K, Puche AC, Shipley MT (2010) Molecular identity of periglomerular and short axon cells. J Neurosci 30:1185-1196. CrossRef Medline

Kosaka T, Kosaka K (2014) Olfactory bulb anatomy. Ref Modul Biomed Sci. Advance online publication. doi: 10.1016/B978-0-12801238-3.04705-X.

Kosaka T, Kosaka K (2016) Neuronal organization of the main olfactory bulb revisited. Anat Sci Int 91:115-127. CrossRef Medline

Lin MZ, Schnitzer MJ (2016) Genetically encoded indicators of neuronal activity. Nat Neurosci 19:1142-1153. CrossRef Medline

Livneh Y, Adam Y, Mizrahi A (2014) Odor processing by adult-born neurons. Neuron 81:1097-1110. CrossRef Medline

Lv X, Zhan C, Zeng S, Chen WR, Luo Q (2006) Construction of multiphoton laser scanning microscope based on dual-axis acousto-optic deflector. Rev Sci Instrum 77:046101. CrossRef

Macrides F, Schneider SP (1982) Laminar organization of mitral and tufted cells in the main olfactory bulb of the adult hamster. J Comp Neur 208:419-430. CrossRef Medline

Madisen L, Zwingman TA, Sunkin SM, Oh SW, Zariwala HA, Gu H, $\mathrm{Ng}$ LL, Palmiter RD, Hawrylycz MJ, Jones AR, Lein ES, Zeng H (2010) A robust and high-throughput Cre reporting and characterization system for the whole mouse brain. Nat Neurosci 13:133140. CrossRef Medline

Mori K, Takahashi YK, Igarashi KM, Yamaguchi M (2006) Maps of odorant molecular features in the mammalian olfactory bulb. Physiol Rev 86:409-433. CrossRef Medline

Nagayama S, Homma R, Imamura F (2014) Neuronal organization of olfactory bulb circuits. Front Neural Circuits 8:98. CrossRef Medline

Najac M, De Saint Jan D, Reguero L, Grandes P, Charpak S (2011) Monosynaptic and polysynaptic feed-forward inputs to mitral cells from olfactory sensory neurons. J Neurosci 31:8722-8729. CrossRef Medline

Najac M, Sanz Diez A, Kumar A, Benito N, Charpak S, De Saint Jan D (2015) Intraglomerular lateral inhibition promotes spike timing variability in principal neurons of the olfactory bulb. $\mathrm{J}$ Neurosci 35:4319-4331. CrossRef Medline

Parrish-Aungst S, Shipley MT, Erdelyi F, Szabo G, Puche AC (2007) Quantitative analysis of neuronal diversity in the mouse olfactory bulb. J Comp Neur 501:825-836. CrossRef Medline 
Pinching AJ, Powell TP (1971) The neuron types of the glomerular layer of the olfactory bulb. J Cell Sci 9:305-345. Medline

Rahmati V, Kirmse K, Holthoff K, Kiebel SJ (2018) Ultra-fast accurate reconstruction of spiking activity from calcium imaging data. $J$ Neurophysiol 119:1863-1878. CrossRef Medline

Raman B, Joseph J, Tang J, Stopfer M (2010) Temporally diverse firing patterns in olfactory receptor neurons underlie spatiotemporal neural codes for odors. J Neurosci 30:1994-2006. CrossRef Medline

Schaefer AT, Margrie TW (2007) Spatiotemporal representations in the olfactory system. Trends Neurosci 30:92-100. CrossRef Medline

Schindelin J, Arganda-Carreras I, Frise E, Kaynig V, Longair M, Pietzsch T, Preibisch S, Rueden C, Saalfeld S, Schmid B, Tinevez JY, White DJ, Hartenstein V, Eliceiri K, Tomancak P, Cardona A (2012) Fiji: an open-source platform for biological-image analysis. Nat Methods 9:676-682. CrossRef Medline

Shao Z, Puche AC, Kiyokage E, Szabo G, Shipley MT (2009) Two GABAergic intraglomerular circuits differentially regulate tonic and phasic presynaptic inhibition of olfactory nerve terminals. J Neurophysiol 101:1988-2001. CrossRef Medline

Shao Z, Puche AC, Liu S, Shipley MT (2012) Intraglomerular inhibition shapes the strength and temporal structure of glomerular output. J Neurophysiol 108:782-793. CrossRef Medline

Spors H, Wachowiak M, Cohen LB, Friedrich RW (2006) Temporal dynamics and latency patterns of receptor neuron input to the olfactory bulb. J Neurosci 26:1247-1259. CrossRef Medline

Tan J, Savigner A, Ma M, Luo M (2010) Odor information processing by the olfactory bulb analyzed in gene-targeted mice. Neuron 65:912-926. CrossRef Medline
Taniguchi H, He M, Wu P, Kim S, Paik R, Sugino K, Kvitsani D, Fu Y, Lu J, Lin Y, Miyoshi G, Shima Y, Fishell G, Nelson SB, Huang ZJ (2011) A resource of Cre driver lines for genetic targeting of GABAergic neurons in cerebral cortex. Neuron 71:995-1013. CrossRef Medline

Tavakoli A, Schmaltz A, Schwarz D, Margrie TW, Schaefer AT, Kollo M (2018) Quantitative association of anatomical and functional classes of olfactory bulb neurons. J Neurosci 38:7204-7220. CrossRef Medline

Theis L, Berens P, Froudarakis E, Reimer J, Román Rosón M, Baden T, Euler T, Tolias AS, Bethge M (2016) Benchmarking spike rate inference in population calcium imaging. Neuron 90:471-482. CrossRef Medline

Uchida N, Poo C, Haddad R (2014) Coding and transformations in the olfactory system. Annu Rev Neurosci 37:363-385. CrossRef Medline

Wachowiak M, Economo MN, Díaz-Quesada M, Brunert D, Wesson DW, White JA, Rothermel M (2013) Optical dissection of odor information processing in vivo using gcamps expressed in specified cell types of the olfactory bulb. J Neurosci 33:5285-5300. CrossRef Medline

Wachowiak M, Shipley MT (2006) Coding and synaptic processing of sensory information in the glomerular layer of the olfactory bulb. Semin Cell Dev Biol 17:411-423. CrossRef Medline

Wellis DP, Scott JW (1990) Intracellular responses of identified rat olfactory bulb interneurons to electrical and odor stimulation. J Neurophysiol 64:932-947. CrossRef Medline

Yaksi E, Friedrich RW (2006) Reconstruction of firing rate changes across neuronal populations by temporally deconvolved $\mathrm{Ca}^{2+}$ imaging. Nat Methods 3:377-383. CrossRef Medline 